\title{
INVESTIGATING THE POTENTIAL OF VISUAL NEWS NARRATIVES TO REDUCE MENTAL ILLNESS STIGMA
}

A Dissertation
presented to
the Faculty of the Graduate School
at the University of Missouri-Columbia
In Partial Fulfillment
of the Requirements for the Degree
Doctor of Philosophy
ROMA SUBRAMANIAN
Dr. Amanda Hinnant, Dissertation Supervisor
December 2016


C Copyright by Roma Subramanian 2016

All Rights Reserved 
The undersigned, appointed by the dean of the Graduate School, have examined the dissertation entitled

INVESTIGATING THE POTENTIAL OF VISUAL NEWS NARRATIVES TO REDUCE MENTAL ILLNESS STIGMA

presented by Roma Subramanian, a candidate for the degree of doctor of philosophy, and hereby certify that, in their opinion, it is worthy of acceptance.

Associate Professor Amanda Hinnant

Associate Professor Kevin Wise

Associate Professor Tim Vos

Professor Glen Cameron

Professor Deborah Hume 


\section{ACKNOWLEDGEMENTS}

I would like to thank my adviser, Dr. Amanda Hinnant, and the rest of the members of my dissertation committee-Dr. Kevin Wise, Dr. Tim Vos, Dr. Glen Cameron, and Dr. Deborah Hume - for their guidance, feedback, and kindness not only during the course of this research but throughout my time in the doctoral program. Many thanks also to Sushma Kumble, Dr. Sungkyoung Lee, Yulia Medvedeva, Dr. Rachel Smith, and Dr. Rachel Young for taking the time to provide thoughtful feedback on the study design and/or data analysis. I am also grateful to the Ellis Library staff and librarians for providing a warm place to work, for access to the Comic Art Collection, and for securing

for me, through interlibrary loans, several graphic novels. A special thanks to Dr. Barbara Gastel, my master's adviser, for continuing to provide mentorship and support.

I would like to acknowledge the Paul Synor Fellowship Program for funding my doctoral studies, which in turn made funding for this dissertation possible. Thanks also to the graduate faculty, the journalism doctoral student body, and the graduate studies office staff (both present and past) for wisdom, friendship, and kindness. Last but not least, thanks to friends and family for encouragement. 


\section{TABLE OF CONTENTS}

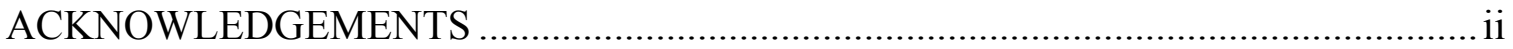

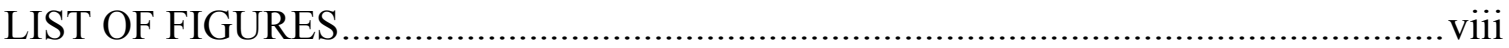

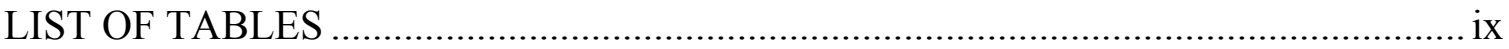

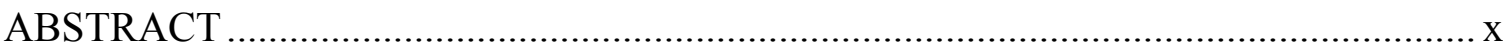

Chapter

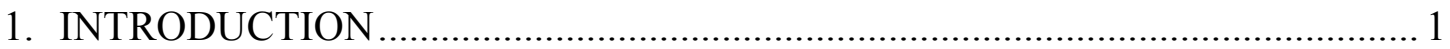

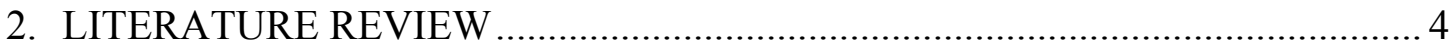

Comics: An Introduction to the Medium

Comics and Health Communication

Audiences' Response to Comics

Comics and Multimedia Learning

Effects of Comics

Effects of Photographs

Stigma and Mental Illness

Stigmatization Process

Types of Stigma

Stigma as a Communication Process

Anti-Stigma Appeals/Communication Messages

Risk Perception and Coping and Stigma

Social Determinants of Health and Stigma

Narratives and Stigma

Nature and Functions of Images

Mental Illness Familiarity and Stigma

Anti-Stigma Behavioral Intention and Behavior 
Political Ideology and Stigma

Images and Learning

3. METHOD ..

Study Design

Participants

Procedure

Stimuli

Text

Images

Photographs

Cartoons

Pre-Test

Dependent Variables

Message Credibility

Identification

Prejudicial Feelings

Pity

Connectedness

Perceived Severity

Perceived Response Efficacy

Perceived Responsibility Beliefs

Social Distance

Coercion-Segregation

Mental-Health Policy Support

Dissemination Likelihood 
Donation

Recall

Manipulation Check

Open-Ended Questions

Moderator Variables

Level of Contact with Mental Illness

Style of Processing

Liberal Ideology

Data Analysis

4. RESULTS

Message Credibility

Perceived Severity and Perceived Response Efficacy

Identification

Affective Reactions

Connectedness

Pity

Prejudicial Feelings

Perceived Responsibility Beliefs

Behavioral Intentions and Behavior

Mental-Health Policy Support

Social Distancing Behavioral Intention

Dissemination Likelihood

Coercion-Segregation Behavioral Intention

Behavior

Interaction Effects with Moderator Political Ideology 
Mental-Health Policy Support

Coercion-Segregation

Interaction Effects with the Mediator Identification

Mental-Health Policy Support

Social Distance Behavioral Intention

Coercion-Segregation

Dissemination Likelihood

Behavior

Interaction Effects with the Mediator Connectedness

Social Distance

Mental-Health Policy Support

Coercion-Segregation

Dissemination Likelihood

Behavior

Analysis of Open-Ended Responses

Depression Condition

Text

Photographs

Humanizing

Cartoons

Illuminating/Illustrative

Inappropriate

Bipolar Disorder Condition

5. DISCUSSION 84

Interpretation of Results 
Depression Condition

Bipolar Condition

Limitations and Future Studies

Theoretical and Practical Significance

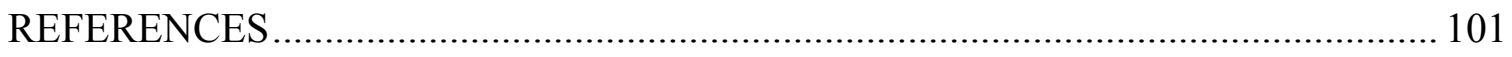

\section{APPENDIX}

1. MODEL RELEASE FORM

VITA 


\section{LIST OF FIGURES}

Figure

Page

1. Wileman's concrete to abstract continuum........................................................ 31

2. Moderation interaction between image type (text/photo) and familiarity with mental illness on dissemination likelihood ............................................................... 74

3. Mediation of the relationship between image type (text/photo) and mental health policy support by identification ................................................................... 76

4. Mediation of the relationship between image type (text/photo) and dissemination

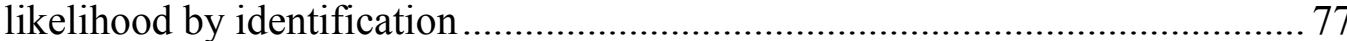

5. Mediation of the relationship between image type (text/photo) and social distance

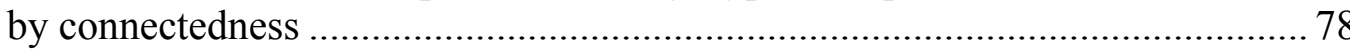

6. Mediation of the relationship between image type (text/photo) and mental health

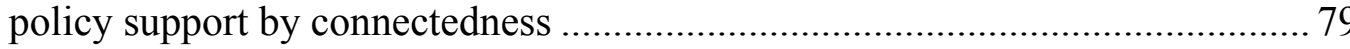

7. Mediation of the relationship between image type (text/photo) and dissemination likelihood by connectedness 


\section{LIST OF TABLES}

Table

Page

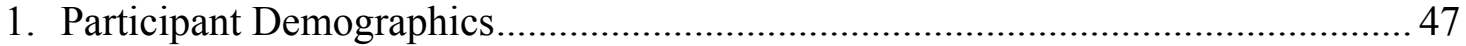




\title{
INVESTIGATING THE POTENTIAL OF VISUAL NEWS NARRATIVES TO REDUCE MENTAL ILLNESS STIGMA
}

\author{
Roma Subramanian
}

Dr. Amanda Hinnant, Dissertation Supervisor

\begin{abstract}
Nearly $50 \%$ of individuals in the United States will develop at least one mental illness during their lifetime (Reeves et al., 2011). Although mental illness can be treated, most people with mental illness do not seek treatment (Phelan, Link, Stueve, \& Pescosolido, 2000). Stigma against mental illness is considered to be a major obstacle to treatment and recovery (USDHHS, 1999). Narratives have the potential to reduce mental illness stigma (for example, Oliver et al., 2012; Chang, 2008). This study extends work on the persuasive potential of narratives by investigating the effects of a particular type of visual narrative on reducing mental illness stigma: Comics. Comics are commonly used in health communication messaging and are believed to offer cognitive and emotional benefits.

Drawing on the stigma communication model, multimedia learning theory, attribution theory, risk perception, and visual persuasion as well as the concept of social determinants of health, a 3 (image type: cartoon, photo, text) x 2 (disease type: depression, bipolar disorder) between-subjects online experiment was conducted to determine whether narrative news stories about mental illness illustrated with abstract/interpretational images (specifically, cartoons, which are a key characteristic of comics) are more effective at reducing mental illness stigma than those illustrated with realistic/representational images, specifically, photographs. Results indicated that for the
\end{abstract}


depression narratives, photographs elicited significantly more anti-stigma behavioral intentions, such as support for mental health policy, likelihood to share the message, and decrease in social distancing behavior, than text alone; further, these effects were mediated by identification and/or connectedness with the story's protagonist. Also, for the depression condition, mean values for the cartoon condition were not significantly lower than those for the photograph condition. For the narratives on bipolar disorder, there were no significant differences between the conditions for any of the outcome variables; however, when it came to empathic response - identification, pity, connectedness - the mean values for the cartoon condition were the highest.

The study contributes theoretically to work on the use of narratives in stigma communication as well as provides practical implications for the use of comics in health communication messages. 


\section{Chapter 1: Introduction}

Nearly $50 \%$ of individuals in the United States will develop at least one mental illness during their lifetime (Reeves et al., 2011). Mental illness is the collective term for “diagnosable mental disorders." These are disorders "characterized by sustained, abnormal alterations in thinking, mood or behavior associated with distress and impaired functioning." Mental illness is also considered a public health problem. In developed countries, mental disorders cause more disability than any other group of disorders, including cancer and heart disease. Although treatment for mental illness is available, most people with mental illness do not seek treatment (Phelan, Link, Stueve, \& Pescosolido, 2000; USDHHS, 1999). Stigma against mental illness is considered to be a major obstacle to treatment and recovery (USDHHS, 1999).

The potential of narratives to reduce stigma against mental illness has been noted (for example, Oliver et al., 2012; Chang, 2008). This study aims to extend work on the persuasive potential of narratives by investigating the effects of visual narratives on reducing mental illness stigma. Specifically, the effects of a particular type of visual narrative are examined: comics. Comics can be defined as "a narrative form consisting of pictures arranged in sequence" (Varnum and Gibbons, 2001; p. xvi) (Tatalovic, 2009).

Comics are believed to offer many advantages in communicating health issues. For example, through their story-telling format, comics can help readers identify with the characters in the story, and in doing so, help them come to terms with their own fears about a particular illness or medical procedure (McNicol, 2014). Because they are perceived as a "non-threatening medium" (McAllister, 1992) that can "universali[ze] the 
illness experience," (Green \& Myers, 2010) comics can be used to foster empathy. Finally, the "interplay of text and image" (page 54) in comics, is believed to make the content more memorable (McNicol, 2014).

However, whether these hypothesized emotional and cognitive benefits are true is not clear. There is not much empirical research on the persuasive effects of comics on readers' cognition, affect, and behavior. Given that comics have long been used in health communication (Schneider, 2014) and continue to be a popular medium in health education (for example, Medikidz comics, a children's comic book series about medical conditions), it is worthwhile to investigate their effects on audiences.

In this study, I aim to fill this gap by examining the effects of comics in communicating psychological disorders. Specifically, drawing on multimedia learning (because a comic is a multimedia material made up of images and text), attribution theory, risk perception, and visual persuasion as well as the concepts of social determinants of health and stigma, I examine how manipulating the nature of the imagerepresentational/realistic, e.g., photographs versus abstract/interpretational, e.g., cartoons - accompanying a narrative news story on mental illness influences various stigma-related outcome variables. Abstract and realistic images have different persuasive effects. For example, the abstract images in comics—cartoons-amplify meaning through their simplicity and lack of details. These simplistic images help readers identify with story characters. On the other hand, realistically drawn characters are believed to "objectify" the characters and in doing so, emphasize "their otherness from the reader" (McCloud, 1993; page 44). Also, by helping readers grasp the essence of a concept, abstract pictures are believed to enhance learning to a greater extent than realistic 
pictures. In this study, abstract images are operationalized as interpretational imagesthey are simplified representational images, analogical images, or arbitrary/logical images, for example, flowcharts, tree diagrams; realistic pictures are operationalized as representational images: they are photorealistic — they resemble the object they represent.

The study examines the effect of image type on outcome variables such as stigmatizing beliefs, emotions, behavioral intentions, and behaviors. I also examine how identification with and empathic response to the story's protagonist mediate these responses. Finally, I examine how individual difference variables such as style of information processing, personal experience with mental illness, and political ideology moderate these effects.

This study contributes to research on multimedia learning by empirically investigating the hypothesized educational and emotional benefits of comics. Given that comics can be described as visual narratives, the study also contributes to research on narratives by applying the principles of narrative persuasion to the medium of comics. Finally, by examining the different components of a comics story - the nature of the text, the nature of the image, and the nature of the reader/learner-in the context of mental illness, the study provides insight into how comics can best be designed to combat stigma against this group of disorders. 


\section{Chapter 2: Literature Review}

\section{Comics: An Introduction to the Medium}

The word "comics" typically conjures up images of children's magazines filled with illustrated fanciful stories about superheroes. However, comics come in various different forms, such as "comic strips, comic books, graphic novels, single-panel cartoons, wordless comics, animated cartoons and various other kinds of visual texts," and can feature humorous or serious content (Tatalovic, 2009, p. 2-3; Varnum \& Gibbons, 2001). It is difficult to find a specific definition that encompasses all these different forms (Varnum \& Gibbons, 2001). For some, the defining feature of comics is the sequential arrangement of pictures. In his seminal book "Understanding Comics," author Scott McCloud defines comics as a medium composed of "juxtaposed pictorial and other [non-pictorial] [static] images in deliberate sequence, intended to convey information and/or to produce an aesthetic response in the viewer" (McCloud, 1993; p. 9). Another definition is "a form of sequential art, often in the form of a strip or a book, in which images and text are arranged to tell a story" (Eisner, 2008; p. 17). Comics have also been defined as "a narrative form consisting of pictures arranged in sequence" (Varnum and Gibbons, 2001; p. xvi) (Tatalovic, 2009). Other common terms for comics that allude to this "sequential" characteristic include "graphic narratives," which encapsulates the nonanimated visual and story components of comics and bande dessinee, meaning "drawings in row" (Petersen, 2011; p. xv). For some, it is not the sequential arrangement of pictures, but the "juxtaposition of words and pictures. ... that is essential to the comics form" (Gibbons \& Varnum; pxvi, as cited in Tatalovic, 2009, p. 2). Based 
upon their length and complexity, Tatalovic (2009) orders different forms of comics in an ascending fashion as follows: "single-frame cartoons, short comic strips, comic books, and graphic novels [which are long-form standalone comics]" (p. 3).

Comics are primarily a visual medium — storytelling depends on "universally understood images, crafted with the intention of imitating or exaggerating reality" (Eisner, 2008; Foreword). However, there is also the view that "art and text" in comics are "equivalent, each driving the other, rather than the illustrations supporting or attempting to explain the text" (Gluibizzi, 2007; p. 28, as cited in Nyberg, 2010, p. 35); in comics, "meaning is produced by the interaction of image and text" (Williams, 2005; p. $6)$.

Comics are characterized by cartoons - simplistic, abstract, pictorial images that are stripped of details, and thereby, amplified in meaning (McCloud, 1993). This amplification is achieved by the cartoon's ability to "focus our attention on an idea" (McCloud, 1993; p. 31), and the simplistic images elicit emotion, specifically, they "provoke identification in the reader" (Squier, 2008; p. 129). Comics artists use realistic pictures to portray the outside world and cartoons to portray the world within (McCloud, 1993; p. 41). Further, "by de-emphasizing the appearance of the physical world in favor of the idea of form, the cartoon places itself in the world of concepts" (McCloud, 1993; p. 41).

Illustrations dominate in comics - words are typically limited to the speech and thoughts of the characters (Hansen, 2004). Another characteristic of comics is that the narrative is fragmented by gaps between frames (Hansen, 2004). These gaps in the narrative are believed to foster engagement by forcing the reader to "participate in 
imagining the story" (Hansen, 2004; p. 155). The "unreal quality of scenery and action" also prompts imagination (Reitberger \& Fuchs, 1972). Finally, comics accelerate the process of text-to-image conversion that occurs when reading text because the images are already provided (Eisner, 2008; p. 17).

Comics have a long history. Although it hard to determine when comics originated, the father of the modern comic strip is believed to be $19^{\text {th }}$ century Swiss painter and schoolmaster Rodolphe Topffer (Petersen, 2010; McCloud, 1993, p. 17). Topffer's picture stories used "cartooning and panel borders, and featured the first interdependent combination of words and pictures seen in Europe" (McCloud, 1993; p. 17).

Comic strips could be found in newspapers as far back as the 1890s (Hansen, 2004). Comic books emerged in the 1930s. "Detective Comics," which appeared in 1937, was a big success, and it was followed by the superhero comic books-Superman (1938) and Batman (1939) (Hansen, 2004). Comic books soon "became a major publishing phenomenon and a multimillion dollar industry" (Hansen, 2004; p. 158). The standard comic book was an eight by ten inch booklet with a glossy cover, printed on newsprint paper, contained different stories, and had graphic art of varied quality (Hansen, 2004).

Besides detective fiction and superhero comics, another genre of comic books that was popular during the early years of the medium were "True Comics," which emerged in 1941. These comics aimed to be educational in that they told stories about real people. A popular story type in this genre was medical histories—stories about scientists and their inventions/discoveries. The popularity of these true-adventure comics began to decline by the mid-1940s (Hansen, 2004). Indeed, the rise of television and concerns 
about the dangers of comic books led to the decline of the medium itself (Hansen, 2004). By the mid-1950s, although comics still had high circulations, they no longer occupied a central place in American popular culture (Hansen, 2004). Over time, the industry began to fragment and subcultures of comic books began to emerge. Comics began to be sold in specialized comic book stores instead of on newsstands. These stores came to be popular among fans of the medium, who were mainly young men.

\section{Comics and Health Communication}

A survey of 254 comics about health from the 1940s to the present found that, over the years, these comics have evolved with regard to "length, topic, and complexity" (Schneider, 2014, p. 996). From educating people, primarily children, about the history of scientific and medical discoveries or providing instruction on how to prevent certain diseases, comics went on to become a tool to change attitudes, for example, changing attitudes toward people with AIDS. Over the past two decades, comics about health have become longer (from about 12 pages in the 1940s and 1950s to more than 100 pages in the 2000s), are more in-depth, targeted at adults, and "are reflective pieces that narrate the author's struggle with physical or psychological disease" (Schneider, 2014; p. 996). These books can be classified as "graphic pathographies"- "illness narratives in graphic form" (Green \& Myers, 2010). These stories have many functions such as helping patients learn about their illness and "providing companionship [with other patients] through shared experience" (Williams, 2011); improving physician-patient communication by humanizing the illness experience, for example, by helping doctors see aspects of patients' personal experience with illness that they may not see in a clinical setting; and facilitating discussion about complex and taboo topics such as sexual abuse 
(Green \& Myers, 2010). Examples of these graphic pathographies include "Mom's Cancer" (Fies, 2006) (about how the author and his family navigate his mother's lung cancer treatment); "Marbles: Mania, Depression, Michaelangelo, and Me" (Forney, 2012) (about how the author comes to terms with her bipolar disorder); and "Years of the Elephant" (Linthout, 2009) (about the author mourning the death of his son by suicide).

\section{Audiences' Response to Comics}

In the past few decades, there has been growing appreciation for comics.

However, in its early days, the medium faced much criticism (Nyberg, 2010). Educators felt that the "one-dimensional characters, the simplistic plots, and the emphasis on the action-adventure story" (Nyberg, 2010; p. 29) would distract children from serious literature, impede their reading ability/act as a barrier to reading skills, and "overexcite young minds with lurid tales of superhero adventure" (Nyberg, 2010; p. 27). Civic and religious leaders felt comics would encourage immorality through their sexual and violent content (Nyberg, 2010; p. 27). Finally, there was concern that comic books could cause psychological damage to children. A key proponent of this fear was psychiatrist Fredric Wertham, who believed that exposure to comic books could lead to juvenile delinquency. Wertham's concerns spurred Senate hearings on the comic book industry in 1954. To safeguard their reputation tarnished by these hearings, comic book publishers formed the trade association the Comics Magazine Association of America. The CMAA adopted a regulatory code to monitor the violent and sexual content in comics. The code also ensured that the "moral messages of comics would strive to cultivate respect for authority and obedience to parents' wishes" (Nyberg, 2010; p. 28). 
In the 1960s and 70s, changes in the reading demographic of comic books from children to young males in their 20 s and 30 s as well as the rise of independent publishers reduced the CMAA's ability to regulate comics content (Nyberg, 2010). Also, attitudes toward comics began to change in the 1970s and 80s (Nyberg, 2010). This has been attributed to the "growing acceptance of mass culture by academia, the refocusing of media effects research on television, and changes in approaches to reading pedagogy" (Nyberg, 2010; p. 30). Today, the comics code no longer exists (Nyberg, 2013).

Academic research has "frequently contradicted the widely held belief that" the language of comics was poor (filled with poor grammar and slang) and reduced reading

ability by encouraging readers to read by relying on the pictures and skipping the text (Nyberg, 2010; p. 28). In recent years, it has come to be realized that readings comics is a complex cognitive task and is not just about making sense of text in word balloons (Nyberg, 2010). In Scott McCloud's words, reading comics requires the reader to be able to "integrate the text and the pictures into an understandable whole" (McCloud, 1993, p. 63). Also, reading comics requires being able to understand the symbolism of imagery (Nyberg, 2010).

\section{Comics and Multimedia Learning}

Comics are believed to facilitate learning in various ways: (1) by making visible abstract phenomena; (2) the juxtaposition of text and image improves learning (the spatial contiguity effect); and (3) the narrative structure of comics facilitates comprehension (Jee \& Angorro, 2012). A useful theory to understand how people process comics is Mayer's cognitive theory of multimedia learning. 
Comics are multimedia materials made up of pictures and text (Kinzer et al., 2012). Mayer (2002), a key figure in research on multimedia learning, defines multimedia messages as "presentations of material using words [which can be written or spoken] and pictures [can be static or dynamic] that are intended to foster learning" (p. 85). According to Mayer, multimedia instruction should foster knowledge construction and not merely information acquisition, that is, a multimedia system should help the learner actively make sense of new information. Mayer defines meaningful learning as the ability to form "coherent mental representations (such as mental models) that are internally organized and externally connected to other knowledge" (p. 101).

Mayer's cognitive theory of multimedia learning is based on three assumptions: (1) the dual-channel assumption: human beings possess separate systems for processing pictorial and verbal (or auditory) information (Paivio, 1986; Baddeley, 1992, as cited in Mayer, 2002); (2) limited capacity assumption: the amount of information that can be processed in each of these channels at one time is limited (Baddeley, 1992, and Chandler \& Sweller, 1991, as cited in Mayer, 2002); in other words, "presenting too much material on the screen at one time can overload the visual/pictorial channel and presenting too much material via the speakers at one time can overload the auditory/verbal channel" (Mayer, 2002; pages 102 and 103); (3) active processing assumption: humans engage in meaningful learning by paying attention to relevant information, organizing this new information in a coherent fashion, and then integrating it with existing knowledge (Mayer, 1999b, Wittrock, 1989).

Mayer's theory of multimedia learning predicts that people learn better when they are presented with information in words and pictures rather than words alone (this is the 
multimedia effect). This corresponds with Paivio's (1986) dual coding theory, according to which memory for an item increases when connections are established between verbal and nonverbal representations of that item.

Although there is support for the multimedia effect (Mayer, 2002), it is important to note, however, that adding pictures to words will not always improve learning; certain conditions must be met for the multimedia effect is to occur (Mayer, 2003). One is the spatial contiguity effect: Text and illustrations can be presented in an integrated manner, that is, words describing the picture embedded in the picture, or in a separated manner, that is, words describing the picture placed on another page.

According to the spatial contiguity effect, the integrated presentation fosters greater learning than the separated presentation. According to the cognitive theory of multimedia learning, the integrated presentation enables learners to "hold corresponding verbal and pictorial representations in working memory at the same time" (p. 109), which facilitates deep learning. On the other hand, in the separated presentation, learners "must expend their limited cognitive resources to search for pictures that correspond to text segments (or vice versa)" (p. 109). Other conditions include the coherence effect-the absence of unrelated material promotes learning — and the personalization effect— learning is more when words are presented in "conversational rather than formal style" (Mayer, 2003, p. 125).

When considering the interaction effects of pictures and text, it's also important to consider the following four variables: "desired performance outcomes" (for example, comprehension, recall, etc.); "nature of the illustrations" (that is, whether the picture is related to the text or not); "nature of the text" (pictures are more helpful with difficult 
text); and "learner characteristics" (pictures are more helpful to learners who lack background knowledge about the text content) (Levin and Mayer, 1993, as cited by Carney and Levin, 2002, p. 7). Other learner characteristics to take into consideration include the learner's age, reading ability, and visual literacy (Peeck, 1993).

Studies on the effects of illustrated text on learning have examined these four variables and how they interact with each other. In their much-cited review of studies ( N $=155)$ that compared learning from illustrated text vs. learning from text alone, Levie and Lentz (1982) found that including pictures with text significantly improved learning of information from both text and pictures; these studies involved both young children and older learners and were conducted using both narrative and expository texts. However, it is important to note that pictures that merely embellish the text (that is, do not overlap with the content of the text or that are not text-redundant) will not improve learning of text information.). The authors conclude that "pictures facilitate the learning of accompanying verbal information that is highly related to the information pictured" (page 213). Also, illustrated text appears to help learning more in poor readers than in good readers. Other conclusions were that the presence of pictures neither helps nor hinders the learning of text content that is not illustrated. Pictures can improve comprehension and delayed recall. Also, in some situations, pictures are more effective than words. Readers may not pay attention to pictures unless they are prompted to do so. Pictures make materials more enjoyable, and finally, learner-generated imagery is typically not as helpful as provided pictures in improving comprehension.

Given that comics are illustrated texts, researchers have attempted to apply the principles of multimedia learning to explain the effects of comics on audiences. 


\section{Effects of Comics}

There is not enough empirical research on the effects of comics (Jee, Angorro, \& Florencia, 2012). The few studies on the effects of comics on learning have found contradictory/mixed results. For example, Liu (2004) investigated whether including comics in English-as-a-second language (ESL) texts of either high- or low-difficulty improves reading and comprehension among high- and low-skilled ESL students. It was found that the comic strip had differential effects on learners of different ESL proficiency. For low-level ESL learners exposed to high-difficulty text, the comics improved their understanding/comprehension of text (as assessed by an immediate recall protocol). However, for high-level ESL learners, the addition of comics to the highdifficulty text did not improve recall of the text. In other words, the addition of comics to text improved learning for the low-level ESL students but not the high-level ESL students. The study results confirm dual-coding theory: the low-level ESL learners benefited when the high-level text was paired with comics because they were able to make sense of the material using two sources of information. But the study also shows that the effects of dual coding theory are not universal. For the high-level learners, the comic did not enhance learning from the text because the comic did not reflect the complexity of the text's linguistic structure. The authors conclude that "visuals that do not reflect the text's linguistic complexity can hinder reading comprehension when the reader assumes that the visual and the text carries the same information" (p. 238-239).

However, a replication of this study conducted by MERC \& Kampusu (2013) with ESL-Turkish learners found that including comic strips in text improves comprehension regardless of student ESL proficiency or text difficulty level. The author 
explains that this conflicting result might be due to differences in the way high- and lowlevel ESL learners were defined in the two studies. On the other hand, Kholi and Forouzesh (2010) found no significant difference between beginning learners of English as a foreign language who were exposed to reading passages with and without comic strips. The authors conclude that for beginning foreign language learners, adding visuals to text does not improve the learners' comprehension. The authors suggest that while selecting visuals, it is important to consider the student's language proficiency level. However, the study has several methodological problems most notable that the students seem to have examined the reading passage and comics in pairs but there is no discussion of how this "group work" might have affected comprehension.

Some studies have looked at the effects of comic books in the context of health and science communication. Here also, the results have been mixed. For example, Lin et al. (2015) examined the effect of a text booklet (only words) versus a comic book on Taiwanese adults' knowledge about and attitude toward nanotechnology as well as their emotions about learning science. No significant difference between the groups was found with regard to these three variables. The authors conclude that the effectiveness of comics is comparable to that of text with regard to science communication. Another study about the effects of comics on science learning found different results. Hosler and Boomer (2011) compared the effectiveness of a comic textbook called "Optical Allusions" on science majors vs. non-science majors. Specifically, the authors examined the effect of the comic book on students' attitude toward biology and attitude toward comics. They also examined the effect of the comic book on knowledge. The subjects were high-school and college students in four different biology classes (sensory biology, biology II, organic 
evolution, and neurobiology) who were taught about vision and evolution using the comic book. There was a significant increase in the median knowledge scores in the four classes; this showed that the comic book did not decrease learning. Also, it was found that "nonmajors' content scores and attitudes showed a statistically significant improvement after using the comic book, particularly among those with lower content knowledge at the start of the semester" (p. 309). Also, this increase in attitude toward biology was correlated with an increase in attitude toward comics. However, a major limitation of the study is lack of a control group that received the same instruction but without comics. Also it is not clear if the non-majors attitude improved because of the comic or because of the narrative in the content. Finally, the study was not a controlled experiment. Students were not randomly assigned to the four biology classes and also students did not have similar demographics.

In the context of health communication, the few studies about the effects of comics have found that this medium is effective. For example, Delp and Jones (1994) conducted a study in which patients with lacerations were given written instructions to care for their wounds. For half the patients, the text instructions were accompanied by illustrations (in cartoon format) that explained the information in the text. It was found that the patients who received the combination of text and illustrations were more attentive to the information and also recalled more information than the other group. However, not all studies about health comics have included control groups. Some such as a study by Sim et al. (2014), who found that comics improve understanding of hepatitis B serology among health professionals, and Sinha et al. (2011), who found that comics increase immediate recall of burn safety methods among first-graders, used pre- and post- 
tests to evaluate gains in knowledge. This makes it difficult to attribute with certainty the learning outcomes to the comics treatment/intervention.

There is need for more work on the individual and interaction effects of the elements involved in processing comics (that is, text, picture, and reader characteristics) to get a better understanding of the conditions under which this medium works best. This is especially important given that comics are a popular medium to communicate health issues. Questions that need to be addressed include how do the nature of the illustrations and text; the placement of text and pictures with regard to each other; as well as the nature of the reader impact processing of comics? Also, what is the effect of comics on outcomes other than learning such as emotions, attitudes, and behavior?

In this study, as a first step toward understanding the effects of comics on audiences, I focus on examining the effects of a unique characteristic of comics, namely, cartoons. Specifically, I examine how narrative news stories about mental illness illustrated with cartoons (simplified, abstract, interpretational pictures) compared with those illustrated with photographs (detailed, realistic, representational pictures) impact mental illness stigma. Before explaining the details of the experiment, I provide a brief look of the use of photographs in news stories.

\section{Effects of Photographs}

Photographs draw readers' attention to the text and also results in them spending more time reading the text (Zillman, Knobloch, \& Yu, 2001). The nature of the photographic images also has an influence on time spent on reading the text. Specifically, the increase in reading time by agonistic images (images that signal danger to self or others, for example, images of people suffering) is about twice that by innocuous images 
(Zillman, Knobloch, \& Yu, 2001).

Studies have found that the addition of relevant images to text increases the recall of information. For example, photographs, particularly emotional ones, increase the recall of newspaper reports (Wanta and Roark, 1993). Also, it has been found that readers recall illustrated newspaper reports better than nonillustrated ones (David, 1998).

Photographs can also influence the interpretation of the story (Zillman, Knobloch, $\& \mathrm{Yu}, 2001)$. For example, large photographs that dominate the page increase the perceived importance of the issue covered in the story (Wanta, 1988). It has also been found that a large picture increases both recall and comprehension of the accompanying story (Huh, 1993). Also, Gibson and Zillman (2000) found that photographs can erroneously exaggerated risk perception. Additionally, photographs that are descriptive and non-arousing and that exemplify only one side of an issue described in a news story produce a biased perception of the issue in the direction of the photograph (Zillman, Gibson, \& Sargent, 1999).

Photographs also increase the credibility of a story as readers have come to believe that photos offer authentic, true accounts of events. News photos "convey a concrete believability that words do not" (Lowrey, 1998, as cited in Greer and Gosen, 2002, p. 5). However, it appears that photographs do not significantly increase the credibility of a news story compared to other types of images. For example, Gruber and Dickerson (2012) examined the effects of different types of images placed above a news story about neuroscience. Subjects were randomly assigned to four groups: For one group, the story was accompanied by an fMRI brain scan image; for another group, the story was accompanied by a non-scientific image--an "artist's rendering of a futuristic 
human head" (p. 944); for a third group, the story was also accompanied by a nonscientific image - an image from a science fiction film. A fourth group, a control group, saw just the story without any image. It was found that there were no significant differences in either evaluations of credibility or scientific reasonableness of the story between the groups.

Based on the above, the following hypotheses are proposed. H1: Participants exposed to news narratives about depression illustrated with images (whether photos or cartoons) will report more perceived story credibility than participants exposed to text-only news narratives.

H2: Participants exposed to news narratives about bipolar disorder illustrated with images (whether photos or cartoons) will report more perceived story credibility than participants exposed to text-only news narratives.

Given that comics are not perceived as a serious form of literature, it is possible that stories illustrated with cartoons compared to those illustrated with photographs will be perceived as less credible.

RQ1: Will participants exposed to a news narrative about depression illustrated with cartoons report significantly less perceived story credibility compared to participants exposed to a news narrative illustrated with photographs?

RQ2: Will participants exposed to a news narrative about bipolar disorder illustrated with cartoons report significantly less perceived story credibility compared to participants exposed to a news narrative illustrated with photographs? 
The news narrative used in the experiment is about mental illness as the study aims to investigate whether visual narratives can reduce mental illness stigma.

\section{Stigma and Mental Illness}

The word "stigma" can be traced to the Greeks and is used to refer to the marks that the Greeks would brand into the skins of people such as criminals and slaves to mark them as "tainted or immoral" (Bos et al., 2013, p. 1). Stigma was originally defined by sociologist Erving Goffman as an attribute that is deeply discrediting and that reduces the bearer "from a whole and usual person to a tainted, discounted one" (Goffman, 1963, p. 3). Stigma can be broadly defined as an undesirable visible or invisible attribute that indicates a spoiled social identity and results in social disapproval (Bos et al., 2013). It is believed that people stigmatize for 3 main reasons: (1) to exploit/dominate, that is, to keep people down; (2) to enforce social norms, that is, to keep people in; and (3) to avoid disease, that is, to keep people away (Phelan, Link, and Dovidio, 2008).

Stigmatizing conditions can be characterized by their dimensions: concealability, course, disruptiveness, aesthetic qualities, origin, and peril (Jones et al., 1984). These dimensions offer a way to distinguish stigmatizing conditions from one another (Link \& Phelan, 2013). Concealability refers to whether a stigmatized condition is visible or not (Goffman, 1963). People with visible stigmas such as race or physical disability are discredited whereas those with concealable stigmas such as mental illness or HIV infection are discreditable (Goffman, 1963). Whether a stigma is concealable or not may not affect the frequency of stigmatizing events that an individual experiences but influences the effectiveness of his/her coping strategies (Chaudoir et al., 2013). 
Course refers to the degree to which a stigmatizing condition is considered to be reversible (Link \& Phelan, 2013). Disruptiveness refers to the extent the condition disrupts or strains interpersonal relationships. Aesthetics refers to the degree to which “different marks elicit an instinctive and affective reaction of disgust" (Link \& Phelan, 2013, p. 532). The more unaesthetic a condition is perceived to be, the more it is stigmatized. Origin is connected to the dimension of "controllability" or attribution. Individuals believed to have caused their stigmatizing condition, for example, substance abuse or obesity, receive more negative responses from others (Link \& Phelan, 2013). On the other hand, when people attribute a low level of responsibility toward an individual for his/her stigmatized condition (for example, breast cancer), they are more likely to express sympathy toward the individual (Bos et al., 2013). Finally, the more a condition is perceived to be frightening (or dangerous), the more stigma it arouses. With regard to mental illness, the fear that individuals with psychosis will harm other people produces a desire for social distance (Link \& Phelan, 2013).

\section{Stigmatization Process}

Link \& Phelan $(2011,2013)$ offer a conceptualization of the stigma process, which has been widely cited. What distinguishes Link and Phelan's (2001) conceptualization of stigma from previous definitions of the concept (for example, by Goffman (1963) and Jones et al. (1984) is that they describe stigma as a social process that is “created by structural power" (Yang et al., 2007, p. 2).

The components of the stigmatization process are briefly described here. First, there is the identification and labeling of human differences that are considered socially relevant or important, such as gender, skin color, IQ. (It is important to note that what 
differences people give salience to are influenced by social, cultural, and economic forces and will vary by time and place.) The labeling or categorization process involves considerable oversimplification. For example, individuals are described as white or black even though there is considerably more variation in human skin color than those categories capture.

In the second step, labeled human differences are linked to undesirable characteristics or attributes - stereotyping. For example, it has been found that people associate or link the label "mental patient" with stereotypes about the dangerousness of the mentally ill. Stereotyping occurs in an automatic manner and is believed to facilitate quick decision-making. Other stereotypes about the mentally ill are that they are incompetent, dirty, and untrustworthy (Link \& Phelan, 2013).

Associating labeled differences with negative characteristics leads to the belief that negatively labeled individuals [them] are "fundamentally different" from people without that label [us] and promotes the poor treatment of labeled individuals. For example, individuals with schizophrenia are often called "schizophrenics," reflecting that these people are not individuals who happen to have a serious disease but that these people are their disease and are therefore fundamentally different from us.

The emotional responses of the stigmatized and stigmatizer are also an important component of the stigmatization process (Link \& Phelan, 2013). For example, the stigmatizer may express negative emotions such as anger, pity, and fear toward a stigmatized person, who in turn may feel embarrassment, shame, or fear (Link \& Phelan, 2013). Shame is a particularly important emotion in stigma process and can have harmful consequences for the stigmatized (Link \& Phelan, 2013). 
Labeling and stereotyping lead to the labeled person experiencing a loss of status and individual and structural discrimination. Finally, it is important to remember that it takes "social, economic, and political" (Link \& Phelan, 2001, p. 375;) power to stigmatize. For example, although it is possible that mentally ill patients in a hospital may identify differences in their treatment providers, label these differences, link these labels with negative characteristics, and treat the providers in a derogatory manner, the providers would not end up experiencing status loss and discrimination because they have more social, economic, and political power than their patients.

To summarize, "stigma exists when elements of labeling, stereotyping, separation, status loss, and discrimination occur together in a power situation that allows them to do so" (Link \& Phelan, 2001, p. 377).

\section{Types of Stigma}

Pryor and Reeder (2011) proposed a model to explain that stigmatization occurs at various levels: self-stigma, stigma by association, structural stigma, and public stigma. Public stigma comprises stigmatizing reactions that arise from how people (perceiver) perceive the features/dimensions of a stigmatized condition (target) and can be explicit or implicit (Bos et al., 2013). Self-stigma broadly refers to how "individuals respond to possessing a stigma" (Chaudoir et al., 2013, p. 76). Stigma by association or courtesy stigma refers to the stigmatizing reactions people express toward individuals associated with a stigmatized person and also "people's reactions to being associated with a stigmatized person" (Bos et al., 2013, p. 2). Finally, structural stigma or discrimination refers to how stigma is perpetuated by "society's institutions and ideological systems" (Pryor \& Reeder, 2011). 


\section{Stigma as a Communication Process}

Rachel Smith (2007) recently proposed a model to describe what are the essential characteristics of stigma communication. Smith's model is evocative of Link \& Phelan's conceptualization of the stigma process. Smith defines stigma communication as messages that "spread through communities to teach their members to recognize the disgraced (i.e., recognizing stigmata) and to react accordingly" (p. 464).

According to Smith's model, stigma messages have 4 characteristics/attributes. These messages contain content cues to "(1) distinguish people and (2) to categorize them as a separate social entity" (3) to link them with social and physical peril and (4) to imply that they are responsible for their condition.

The content cues trigger cognitive (the activation of stereotypes) and emotional reactions (such as anger, fear, and disgust)—-both of which foster stigmatizing attitudes. People who are exposed to such stigma messages may then want to share them with others because these messages encourage "in-group solidarity."

\section{Anti-Stigma Appeals/Communication Messages}

Based on Smith's model, it can be proposed that by corollary, anti-stigma messages should contain content cues that (1) instead of distinguishing the stigmatized, increase identification with individuals with a stigmatized disease and thereby decrease the perceived entiativity of the stigmatized group; (2) demonstrate stigmatized individuals' social value/moral value/how they practice reciprocity/that the stigmatized do not hinder the functioning of the group; (3) demonstrate how stigmatized individuals are at the receiving end of social and physical peril and are not the cause of it/ demonstrate that stigmatized individuals are not responsible for their condition/indicate 
external attribution for their condition; and (4) encourage collective efforts to eliminate the stigma.

The above definition of an anti-stigma appeal targets stigma at both the individual and structural level. It's important that anti-stigma messages do so because stigma is context-dependent-it is rooted in interpersonal relationships and is influenced by the culture and structure of society. So, a large number of individual-level-directed interventions, no matter how successful, will continually confront "negative reinforcement from the larger culture" (Pescosolido et al., 2013).

Another reason to consider targeting both individual and structural levels of stigma is that the mechanisms underlying these processes may be different. For example, with regard to mental illness, it has been found that individual discrimination (that is, social distance) is related to perceptions of fear and dangerousness of the mentally ill; however, structural discrimination is related to attributions of responsibility for the disease (Angermeyer \& Matschinger, 2014).

This study investigates the effect of an anti-stigma appeal in the form of narrative news stories about mental illness. Specifically, the study examines how the nature of the images/visuals accompanying the narrative influences stigmatizing responses.

\section{Risk Perception and Coping and Stigma}

In the context of physical illness, studies have found that the use of visuals to communicate health information increases risk perceptions (Lee, Cameron, Wunsche, and Stevens, 2011). Chang (2013) provides a brief review of these studies. For example, with regard to public service announcements on sun protection, Boer, Huurne, and Taal (2006) found that people exposed to textual + pictorial information recalled more information 
about the negative effects of sun exposure than those exposed to just textual information. With regard to health news, Gibson and Zillman (2000) found that when people were exposed to a news story about a fictitious disease, their risk perception was lower if the story was not accompanied with pictures. According to Zillman and Brosius (2000), pictures increase risk perception by increasing the vividness of risk information, and thereby making that information more accessible. In addition to enhancing risk perception, pictures enhance efficacy to cope with the risk by, for example, helping people imagine coping behaviors and by illustrating the processes involved (Chang, 2013) and by helping to illustrate that the coping behaviors are effective.

The symptoms of mental illness are not always visible and can be hard to imagine (for example, hallucinations). It can also be hard to comprehend how to cope with a mental illness. One of the advantages of the comics medium is that they can make visible invisible or abstract phenomena. Illustrations of symptoms of mental illness might help make them more vivid, and in doing so, also increase perceived severity of the illness. Illustrations may also enhance perceived response efficacy, which refers to perceptions of the effectiveness of the proposed strategies to prevent or treat the illness (Witte, 1992).

Based on the above, the following hypotheses are proposed:

H3: Participants exposed to a news narrative about depression illustrated with images (whether photos or cartoons) will report more perceived disease severity than participants exposed to a text-only news narrative.

H3a: Participants exposed to a news narrative about depression illustrated with cartoons will report more perceived disease severity than participants exposed to a news narrative illustrated with photos. 
H4: Participants exposed to a news narrative about bipolar disorder illustrated with images (whether photos or cartoons) will report more perceived disease severity than participants exposed to a text-only news narrative.

H4a: Participants exposed to a news narrative about bipolar disorder illustrated with cartoons will report more perceived disease severity than participants exposed to a news narrative illustrated with photos.

H5: Participants exposed to a news narrative about depression illustrated with images

(whether photos or cartoons) will report more perceived response efficacy than participants exposed to a text-only news narrative.

H5a: Participants exposed to a news narrative about depression illustrated with cartoons will report more perceived response efficacy than participants exposed to a news narrative illustrated with photos.

H6: Participants exposed to a news narrative about bipolar disorder illustrated with images (whether photos or cartoons) will report more perceived response efficacy than participants exposed to a text-only news narrative.

H6a: Participants exposed to a news narrative about bipolar disorder illustrated with cartoons will report more perceived response efficacy than participants exposed to a narrative illustrated with photos.

In addition to risk perception, another variable that influences stigmatizing responses is how responsibility for health outcomes is configured.

\section{Social Determinants of Health and Stigma}

Social determinants of health have been defined as including "daily living conditions and the distribution of power, money and resources" (Todman, 2011). The 
social determinants of mental health have been described as adverse social conditions that contribute to triggering chronic stress (particularly in individuals who are genetically predisposed to stress sensitivity), which can increase the risk of mental illness (Fisher \& Baum, 2010). Examples of these social conditions include "low income, social isolation, insecure housing, unemployment, living in an unsafe community, and gendered violence" (Fisher \& Baum, 2010, p. 1058). It has been found that stigma can be reduced by shifting attributions of responsibility for both the cause and solution of a health problem away from an individual and toward society by raising awareness about the social determinants of health.

According to attribution theory, people attribute the cause of an event to either internal or external factors (Weiner et al., 1988). Attributing an issue to internal factors reflects the belief that "a person's disposition is caused by that person's characteristics and so is within that person's control" (Niederdeppe et al., 2008, p. 487). On the other hand, attributing an issue to external factors reflects the belief that "a person's disposition is caused by contextual factors and so is outside that person's control" (Niederdeppe et al., 2008, p. 487). For example, poverty can be attributed to internal factors - being lazy and lacking in motivation—or external factors—living in a destitute neighborhood (Niederdeppe et al., 2008, p. 487).

According to Weiner $(1993,2006)$, “(a) beliefs about what causes a social problem ([internal or external] causal attributions) influence (b) beliefs about who is responsible for addressing it ([internal or external] solution attributions), which in turn shape (c) personal behaviors (personal actions to help others) and support for proposed governmental solutions (policy beliefs) to address the problem" (Niederdeppe et al., 
2011, p. 297). With regard to stigmatized health conditions, individuals believed to have caused their stigmatizing condition, for example, substance abuse or obesity, receive more negative responses from others (Link \& Phelan, 2013). On the other hand, when people attribute a low level of responsibility toward an individual for his/her stigmatized condition (for example, breast cancer), they are more likely to express sympathy toward the individual (Bos et al., 2013).

Understanding the social conditions or social circumstances that lead people to be exposed to individual-level risk factors for a disease helps to get at the root or fundamental causes of a disease. For example, encouraging people to change unhealthy habits (for example, eating fatty food) without understanding the social forces that lead them to engage in those unhealthy habits (for example, lack of transportation to access fresh vegetables) puts the burden of responsibility for safeguarding health on the individual and does little to change the fundamental social factors that put people at risk for disease (Link \& Phelan, 1995). Therefore, it is important to pay attention to how cause and responsibility for health issues are framed in news coverage. Contextualizing the origin of individual-level risk factors for a disease can deflect responsibility for managing the disease away from the individual and toward society.

Two strategies suggested to increase salience of social determinants of health are narratives and visual imagery (Niederdeppe et al., 2008). Given that comics are visual narratives, I now turn to a brief discussion of how narratives work and how narrative persuasion relates to stigma reduction. 


\section{Narratives and Stigma}

A narrative is "any cohesive and coherent story with an identifiable beginning, middle, and end that provides information about scene, characters, and conflict; raises unanswered questions or unresolved conflict; and provides resolution" (Hinyard \& Kreuter, 2007, p. 778).

The persuasive potential of narratives is well-known. It has been shown that narratives have the potential to change peoples' beliefs and attitudes through the mechanism of transportation (Green \& Brock, 2000). Narrative transportation is defined as "a distinct mental process, an integrative melding of attention, imagery, and feelings" (Green \& Brock, 2000, p. 701). Transportation is the "degree to which an individual is cognitively and affectively invested while reading a narrative" (Caputo \& Rouner, 2011, p. 596). With regard to news on mental illness, a recent study found that compared to news stories in a non-narrative format, narrative stories were more effective at inducing transportation (Oliver et al., 2012).

Narratives also lead to more identification (Cohen, 2001). Identification has been defined as "as an imaginary process that entails merging with the character and sharing the character's knowledge about the narrated events, adopting the character's goals (i.e. hoping that they succeed), and sharing the character's emotions (Tal-Or \& Cohen, 2010, p. 404; Cohen, 2001, 2006). Identification is distinct from transportation in that while transportation refers to engagement with the narrative as a whole, the experience of identification is restricted to a specific character in the narrative (Tal-Or \& Cohen, 2010).

Narratives also trigger positive emotional responses. A study also found that the narrative stories were more effective at inducing compassion toward stigmatized 
individuals, by encouraging readers to take the perspective of these individuals, and also favorable behavioral intentions toward these individuals (Oliver et al., 2012). Another study showed that narrative ads on depression generated more sympathy toward depressed individuals than non-narrative ads (Chang, 2008). It has also been found that the more relevant participants find a narrative on mental illness, the lower is the social distancing behavior (a measure of stigma) they demonstrate (Caputo \& Rouner, 2011). Identification with and the emotional response to a story character could be influenced by the nature of the image that accompanies the story. It is worthwhile to examine different forms of images and their function.

\section{Nature and Functions of Images}

Images can be classified into different types depending on their level of realism (Wileman, 1993, as cited by Medley \& Haddad, 2011). Images can be said to exist along a continuum from "concrete at the realistic end to abstract at the distilled end" (Wileman, 1993, cited in Medley \& Haddad, 2011, p. 5). Types of images along this continuum in the order of increasing abstraction are "pictorial symbols, graphic symbols, and verbal symbols" (Wileman, 1993, cited in Medley \& Haddad, 2011, p. 5). Examples of pictorial symbols are photographs and illustrations/drawings that represent a particular object or thing in a highly realistic manner. Graphic symbols are less realistic, and verbal symbols are purely abstract, for example, words. See Figure 1 below for an illustration of this continuum. Abstraction or a decrease in realism "moves images from the particular to the generic; from a focus on surface to a focus on structure; and from mimetic to symbolic" (Rodman, 1985, cited in Medley \& Haddad, 2011, p. 6). 


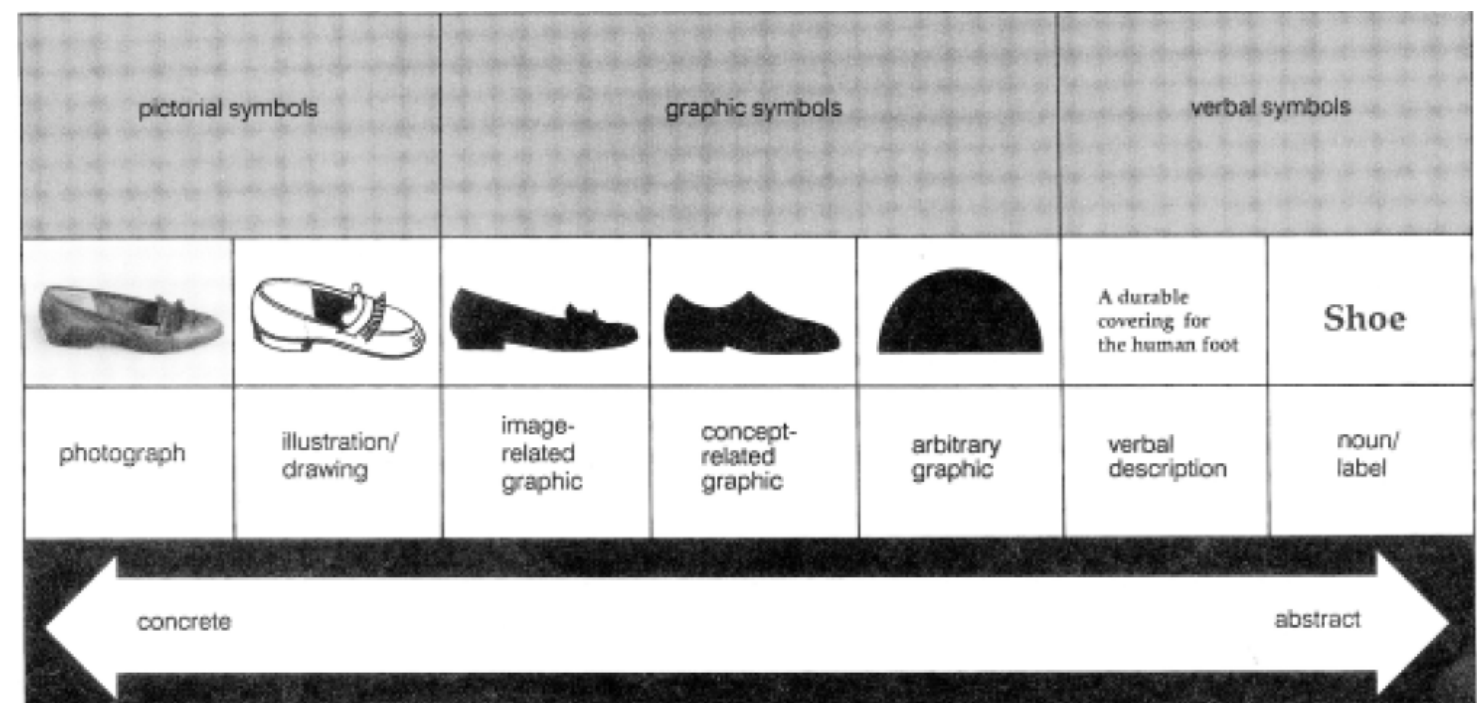

Figure 1: Wileman's concrete to abstract continuum (reproduced from Medley \& Haddad, 2011)

As explained earlier, comics are characterized by cartoons — simplistic, abstract, pictorial images that are stripped of details, and thereby, amplified in meaning (McCloud, 1993). This amplification is achieved by the cartoon's ability to "focus our attention on an idea" (McCloud, 1993, p. 31), and the simplistic images elicit emotion, specifically, they "provoke identification in the reader" (Squier, 2008, p. 129). Comics artists use realistic pictures to portray the outside world and cartoons to portray the world within (McCloud, 1993, p. 41). Further, "by de-emphasizing the appearance of the physical world in favor of the idea of form, the cartoon places itself in the world of concepts" (McCloud, 1993, p. 41). Finally, while simply drawn characters "assist in reader identification" realistically drawn characters "objectify" the characters and in doing so, emphasize "their otherness from the reader" (McCloud, 1993, p. 44).

Based on the above, the following hypotheses are proposed. H7: There will be a significant difference with regard to identification with the story's protagonist between participants exposed to a news narrative about depression 
accompanied with cartoons versus those exposed to a narrative with photos and those exposed to a narrative with text-alone (control).

H8: There will be a significant difference with regard to identification with the story's protagonist between participants exposed to a news narrative about bipolar disorder accompanied with cartoons versus those exposed to a narrative with photos and those exposed to a narrative with text-alone (control).

H9: There will be a significant difference with regard to connectedness with the story's protagonist between participants exposed to a news narrative about depression accompanied with cartoons versus those exposed to a narrative with photos and those exposed to a narrative with text-alone (control).

H10: There will be a significant difference with regard to connectedness with the story's protagonist between participants exposed to a news narrative about bipolar disorder accompanied with cartoons versus those exposed to a narrative with photos and those exposed to a narrative with text-alone (control).

H11: There will be a significant difference with regard to pity for the story's protagonist between participants exposed to a news narrative about depression accompanied with cartoons versus those exposed to a narrative with photos and those exposed to a narrative with text-alone (control).

H12: There will be a significant difference with regard to pity for the story's protagonist between participants exposed to a news narrative about bipolar disorder accompanied with cartoons versus those exposed to a narrative with photos and those exposed to a narrative with text-alone (control).

H13: There will be a significant difference with regard to prejudicial feelings for the 
story's protagonist between participants exposed to a news narrative about depression accompanied with cartoons versus those exposed to a narrative with photos and those exposed to a narrative with text-alone (control).

H14: There will be a significant difference with regard to prejudicial feelings for the story's protagonist between participants exposed to a news narrative about bipolar disorder accompanied with cartoons versus those exposed to a narrative with photos and those exposed to a narrative with text-alone (control).

H15: There will be a significant difference with regard to perceived responsibility beliefs between participants exposed to a news narrative about depression illustrated with cartoons versus those exposed to a news narrative illustrated with photos or those exposed to a narrative with only text (control).

H16: There will be a significant difference with regard to perceived responsibility beliefs (that is, beliefs about how much responsibility for the disease should be attributed to the individual) between participants exposed to a news narrative about bipolar disorder illustrated with cartoons versus those exposed to a news narrative illustrated with photos or those exposed to a narrative with only text (control).

\section{Mental Illness Familiarity and Stigma}

With regard to mental illness stigma, it has also been found that familiarity with mental illness (for example, by having a family member or friend with mental illness) influences the level of social distance toward the mentally ill such that people who have experienced contact with the mentally ill are less likely to harbor stigmatizing attitudes toward them (Corrigan et al., 2001). The expectation is that mental-illness familiarity will intensify the predicted outcomes for H7-H16. 
Based on this, the following hypotheses are proposed:

H7a: There will be a significant difference with regard to identification with the story's protagonist between participants exposed to a news narrative about depression accompanied with cartoons versus those exposed to a narrative with photos and those exposed to a narrative with text-alone (control), and familiarity with mental illness will moderate this effect.

H8a: There will be a significant difference with regard to identification with the story's protagonist between participants exposed to a news narrative about bipolar disorder accompanied with cartoons versus those exposed to a narrative with photos and those exposed to a narrative with text-alone (control), and familiarity with mental illness will moderate this effect.

H9a: There will be a significant difference with regard to connectedness with the story's protagonist between participants exposed to a news narrative about depression accompanied with cartoons versus those exposed to a narrative with photos and those exposed to a narrative with text-alone (control), and familiarity with mental illness will moderate this effect.

H10a: There will be a significant difference with regard to connectedness with the story's protagonist between participants exposed to a news narrative about bipolar disorder accompanied with cartoons versus those exposed to a narrative with photos and those exposed to a narrative with text-alone (control), and familiarity with mental illness will moderate this effect.

H11a: There will be a significant difference with regard to pity for the story's protagonist between participants exposed to a news narrative about depression accompanied with 
cartoons versus those exposed to a narrative with photos and those exposed to a narrative with text-alone (control), and familiarity with mental illness will moderate this effect. H12a: There will be a significant difference with regard to pity for the story's protagonist between participants exposed to a news narrative about bipolar disorder accompanied with cartoons versus those exposed to a narrative with photos and those exposed to a narrative with text-alone (control), and familiarity with mental illness will moderate this effect.

H13a: There will be a significant difference with regard to prejudicial feelings for the story's protagonist between participants exposed to a news narrative about depression accompanied with cartoons versus those exposed to a narrative with photos and those exposed to a narrative with text-alone (control), and familiarity with mental illness will moderate this effect.

H14a: There will be a significant difference with regard to prejudicial feelings for the story's protagonist between participants exposed to a news narrative about bipolar disorder accompanied with cartoons versus those exposed to a narrative with photos and those exposed to a narrative with text-alone (control), and familiarity with mental illness will moderate this effect.

H15a: There will be a significant difference with regard to perceived responsibility beliefs (that is, degree to which individual is responsible for his/her condition) between participants exposed to a news narrative about depression illustrated with cartoons versus those exposed to a news narrative illustrated with photos or those exposed to a narrative with only text (control), and familiarity with mental illness will moderate this effect. H16a: There will be a significant difference with regard to perceived responsibility 
beliefs between participants exposed to a news narrative about bipolar disorder illustrated with cartoons versus those exposed to a news narrative illustrated with photos or those exposed to a narrative with only text (control), and familiarity with mental illness will moderate this effect.

\section{Anti-Stigma Behavioral Intention and Behavior}

Identification with the narrative's protagonist as well as feelings of connectedness with the protagonist should lead to anti-stigma behavioral intentions and behaviors. Specifically, this study looks at whether identification and connectedness reduce social distancing behavior (public stigma) and increase the likelihood of sharing the anti-stigma message (public stigma). Further, the study examines whether identification and connectedness lead to support for mental health policies (structural stigma), a reduction in coercion-segregation behavioral intentions (structural stigma), and willingness to donate to mental health organizations (structural stigma).

Structural stigma is an example of a social determinant of health. Niederdeppe et al. (2008) suggested the use of visual imagery to make salient the social determinants of health. Studies have used images to convey the social determinants of health and have examined responses to these visuals, be it in health campaigns (for example, Young, Subramanian, \& Hinnant, 2015) or in news stories (Niederdeppe et al., 2015).

How does one convey the social determinants of health using images? A type of picture that might be helpful to communicate this complex and abstract concept is analogical pictures (Alessandrini, 1984). As the name suggests, these pictures convey "a concept or topic by showing something else and implying a similarity" (p. 68). They "might be especially useful when the concept or topic to be communicated is abstract or 
has no tangible existence so that direct portrayal is difficult" (p. 68). Studies have found that the use of visual analogies can improve learning in adults (as reviewed by Alessandrini, 1984). Visual metaphors are an integral part of the comics language.

There are also arbitrary or logical pictures. These are "highly schematized visuals [that] do not look like the things they represent but are related logically or conceptually" (Alessandrini, 1984, p.70). Examples of these pictures are graphs, flowcharts, tree diagrams, and maps (Alessandrini, 1984). Studies on the effects of arbitrary pictures have found that they improve learning, and it is believed that they do so by "abstractly portray[ing] the essence of new concepts" (Alessandrini, 1984, p. 73). In this study, realistic images are operationalized as representational images: they are photographsthey resemble the object they represent. Abstract images are operationalized as interpretational images: they are cartoons_-simplified versions of representational images, analogical, or arbitrary.

Based on the above, the following hypotheses are proposed:

H17: There will be a significant difference with regard to mental health policy support between participants exposed to a news narrative about depression illustrated with cartoons versus those exposed to a news narrative illustrated with photos or those exposed to a narrative with only text (control).

H17a: This effect will be mediated by identification with the story character.

H17b: This effect will be mediated by connectedness with the story character.

H17c: This effect will be moderated by familiarity with mental illness.

H18: There will be a significant difference with regard to mental health policy support between participants exposed to a news narrative about bipolar disorder illustrated with 
cartoons versus those exposed to a news narrative illustrated with photos or those exposed to a narrative with only text (control).

H18a: This effect will be mediated by identification with the story character.

H18b: This effect will be mediated by connectedness with the story character.

H18c: This effect will be moderated by familiarity with mental illness.

H19: There will be a significant difference with regard to social distance behavioral intention between participants exposed to a news narrative about depression illustrated with cartoons versus those exposed to a news narrative illustrated with photos or those exposed to a narrative with only text (control).

H19a: This effect will be mediated by identification with the story character.

H19b: This effect will be mediated by connectedness with the story character.

H19c: This effect will be moderated by familiarity with mental illness.

H20: There will be a significant difference with regard to social distance behavioral intention between participants exposed to a news narrative about bipolar disorder illustrated with cartoons versus those exposed to a news narrative illustrated with photos or those exposed to a narrative with only text (control).

H20a: This effect will be mediated by identification with the story character. H20b: This effect will be mediated by connectedness with the story character. H20c: This effect will be moderated by familiarity with mental illness.

H21: There will be a significant difference with regard to message dissemination intention between participants exposed to a news narrative about depression illustrated with cartoons versus those exposed to a news narrative illustrated with photos or those exposed to a narrative with only text (control). 
H21a: This effect will be mediated by identification with the story character.

H21b: This effect will be mediated by connectedness with the story character.

H21c: This effect will be moderated by familiarity with mental illness.

H22: There will be a significant difference with regard to message dissemination intention between participants exposed to a news narrative about bipolar disorder illustrated with cartoons versus those exposed to a news narrative illustrated with photos or those exposed to a narrative with only text (control).

H22a: This effect will be mediated by identification with the story character.

H22b: This effect will be mediated by connectedness with the story character.

H22c: This effect will be moderated by familiarity with mental illness.

H23: There will be a significant difference with regard to coercion-segregation intention between participants exposed to a news narrative about depression illustrated with cartoons versus those exposed to a news narrative illustrated with photos or those exposed to a narrative with only text (control).

H23a: This effect will be mediated by identification with the story character.

H23b: This effect will be mediated by connectedness with the story character.

H23c: This effect will be moderated by familiarity with mental illness.

H24: There will be a significant difference with regard to coercion-segregation intention between participants exposed to a news narrative about bipolar disorder illustrated with cartoons versus those exposed to a news narrative illustrated with photos or those exposed to a narrative with only text (control).

H24a: This effect will be mediated by identification with the story character.

H24b: This effect will be mediated by connectedness with the story character. 
H24c: This effect will be moderated by familiarity with mental illness.

H25: There will be a significant difference with regard to willingness to donate MTurk earnings to mental health organizations between participants exposed to a news narrative about depression illustrated with cartoons versus those exposed to a news narrative illustrated with photos or those exposed to a narrative with only text (control). H25a: This effect will be mediated by identification with the story character. H25b: This effect will be mediated by connectedness with the story character. H25c: This effect will be moderated by familiarity with mental illness.

H26: There will be a significant difference with regard to willingness to donate MTurk earnings to mental health organizations between participants exposed to a news narrative about bipolar disorder illustrated with cartoons versus those exposed to a news narrative illustrated with photos or those exposed to a narrative with only text (control). H26a: This effect will be mediated by identification with the story character. H26b: This effect will be mediated by connectedness with the story character. H26c: This effect will be moderated by familiarity with mental illness.

\section{Political Ideology and Stigma}

Attitudes toward the mentally ill are also influenced by political ideology. For example, it's been found that individuals who are hierarchical and communitarian in their outlook are more likely to support outpatient commitment laws for people with mental illness than those who are egalitarian and individualistic (Kahan et al., 2010). The latter qualities align with liberal identity (Wildavsky \& Dake, 1990). Based on this, the following hypotheses are proposed.

H27: There will be a significant difference with regard to mental health policy support 
between participants exposed to a news narrative about depression illustrated with cartoons versus those exposed to a news narrative illustrated with photos or those exposed to a narrative with only text (control), and this effect will be moderated by liberal ideology.

H28: There will be a significant difference with regard to coercion-segregation intention between participants exposed to a news narrative about depression illustrated with cartoons versus those exposed to a news narrative illustrated with photos or those exposed to a narrative with only text (control)., and this effect will be moderated by liberal ideology.

H29: There will be a significant difference with regard to mental health policy support between participants exposed to a news narrative about bipolar disorder illustrated with cartoons versus those exposed to a news narrative illustrated with photos or those exposed to a narrative with only text (control),, and this effect will be moderated by liberal ideology.

H30: There will be a significant difference with regard to coercion-segregation intention between participants exposed to a news narrative about bipolar disorder illustrated with cartoons versus those exposed to a news narrative illustrated with photos or those exposed to a narrative with only text (control), and this effect will be moderated by liberal ideology.

\section{Images and Learning}

In addition to potentially different influences on identification, realistic versus abstract images have different effects on learning. For example, Mason et al. (2013) investigated how different types of illustrations - concrete/contextualized or 
abstract/decontextualized—influenced learning from a science text. Factual knowledge and transfer (which is being able to apply knowledge to other situations) was measured in an immediate post-test as well as in a delayed post-test. The text was about the forces that act on a body on an inclined plane. In the concrete condition, the inclined plane was depicted by "a snow-covered hillside, and the descending body was a skier" (p. 363). The abstract condition "was a decontextualized representation as the plane and descending body were depicted schematically with no reference to any realistic scenario" (p. 363).

It was expected that both types of illustrated texts would enhance factual knowledge and transfer at immediate post-test compared to the non-illustrated text. It was also expected that the text illustrated with the concrete pictures would enhance factual knowledge to a greater extent than the text illustrated with the abstract pictures at the delayed post-test because concrete illustrations "contain retrieval cues that are beneficial for memory" (Ericsson \& Kintsch, 1995, as cited by Mason et al., 2013, p. 361). In other words, "the information conveyed is made more available in long-term memory by the concreteness of the representation (Koedinger \& Nathan, 2004, as cited by Mason et al., 2013, p. 361). It was also hypothesized that both types of illustrated texts would enhance transfer to a greater extent than the non-illustrated text; further, the abstract illustrated text would enhance transfer to a greater extent than the concrete illustrated text because abstract pictures "help learners focus more on the structural characteristics of a phenomenon or situation (Colin et al., 2002; as cited by Mason et al., 2013, p. 361).

The authors found that the illustrated text—regardless of the type of illustrationimproved learning - to a greater degree than the text alone. Also, at the delayed posttest, the concrete illustrated text produced most learning. However, with regard to knowledge 
transfer, as against expectations, the abstract illustrated text and concrete illustrated text performed equally well. (The authors surmise that this might be because the knowledge transfer test was not strong enough; in other words, subjects were assessed on their ability to apply what they had learned to a pretty similar situation, not a drastically different one. So it's hard to determine whether knowledge transfer was indeed occurring or not.) Also, readers exposed to the text with the abstract illustration were more efficient at text processing and spent more time integrating the picture and the text. The authors conclude that abstract illustrations may be more beneficial for learning concepts whereas concrete illustrations may be more useful for learning facts.

Support for this effect of realistic versus abstract pictures has also been found in more than 100 studies with adult learners conducted by Dwyer (1978; 1982-1983) et al. on the effects of realistic pictures that vary in detail (ranging from color photos to line drawings) (as cited in Alessandrini, 1984). The authors conclude "that simple line drawings are most helpful to learners while overly concrete or detailed visuals, such as photographs, do not aid learning” (p. 68, as cited in Alessandrini, 1984). The authors also found that realistic images do not improve learning if the material is too complex.

Based on the above, the following hypotheses are proposed:

H31: Participants exposed to a news narrative about depression illustrated with cartoons will have higher recall scores than participants exposed to a news narrative illustrated with photos.

H31a: This effect will be greater for those who have a visual rather than verbal style of processing. 
H32: Participants exposed to a news narrative about bipolar disorder illustrated with cartoons will have higher recall scores than participants exposed to a news narrative illustrated with photos.

H32a: This effect will be greater for those who have a visual rather than verbal style of processing. 


\section{Chapter 3: Method}

\section{Study Design}

The study used a 3 (image: photograph, cartoon, no image/control) x 2 (message repetition: news narrative on depression, news narrative on bipolar disorder) betweensubjects design. Depression and bipolar disorder were chosen as representative examples of common and severe mental illness, respectively. A priori power analysis using the G*Power 3.1 statistical power analysis program (Faul, Erdfelder, Buchner, \& Lang, 2009 ) indicated a sample size of 251 for $95 \%$ power to detect a medium-sized effect ( $\mathrm{f}=$ $.25)$ at $\alpha=.05$. To compensate for potentially poor responses and to ensure adequate statistical power, the estimated sample size was increased by about $30 \%$. The final sample size was 320 .

\section{Participants}

A sample of 320 American adults was recruited through the Amazon Mechanical Turk (MTurk) website. MTurk is an online marketplace in which individuals or businesses (called "Requesters") access "to a global, on-demand, 24x7 workforce" (called "Workers”) (“Amazon Mechanical Turk," n.d.). MTurk workers are more representative of the general population; they are also less expensive to recruit (Berinsky, Huber, and Lenz, 2012). However, it is important to note that MTurk workers are typically younger, more politically liberal than the general public, and also pay more

attention to tasks (Berinsky et al., 2012). These factors could influence how they respond to study questions and limit the external validity of the study (Berinsky et al., 2012). However, given that internal validity is the primary concern of experiments, these issues 
are not of serious concern. MTurk has been found to be a useful way to collect data (Behrend et al. 2011; Paolacci et al. 2010; Rand 2012).

Requesters post an assignment or a "human intelligence task" (also called "HIT") on the MTurk website. Workers can view the HIT and then decide to accept or reject it. To ensure good quality data, requesters can control which workers work on their HITs by only permitting workers who meet certain criteria to work on their HITs, for example, workers who have a HIT approval rate of 95\% (approval rate is the percentage of submitted assignments that requesters have approved) and workers who have 100 or more approved HITs (number of HITs approved is the total number of assignments/tasks that have been approved for the worker). After a worker accepts and completes a particular HIT and the requester approves the work, the reward/payment for the HIT is transferred from the requester's MTurk account to the worker's Amazon payment account ("Mechanical Turk Concepts," n.d.).

Sample inclusion criteria for this study were as follows: age, 18 or older; location, United States; HIT approval rate (\%), greater or equal to 95; number of HITs approved, greater or equal to 100. Informed consent was obtained from all participants. Participants were compensated $\$ 2.00$ for participating in the study. Participant demographic information is provided in Table 1. 
Table 1. Participant Demographics.

\begin{tabular}{|c|c|}
\hline Characteristic & No. $(\%)$ of participants $(n=320)$ \\
\hline \multicolumn{2}{|l|}{ Gender } \\
\hline Male & $163(50.6)$ \\
\hline Female & $155(48.1)$ \\
\hline Other & $1(.3)$ \\
\hline Don't want to say & $1(.3)$ \\
\hline \multicolumn{2}{|l|}{ Age (years) } \\
\hline $\mathrm{M}(\mathrm{SD})$ & $33.4(9.62)$ \\
\hline Range & $19-77$ \\
\hline \multicolumn{2}{|l|}{ Race } \\
\hline Black/African-American & $23(7.1)$ \\
\hline White & $253(78.6)$ \\
\hline Latino/Hispanic & $13(4)$ \\
\hline Asian & $24(7.5)$ \\
\hline Other & $5(1.6)$ \\
\hline Don't want to say & $2(.6)$ \\
\hline \multicolumn{2}{|l|}{ Marital status } \\
\hline Married/Living with partner & $155(48.1)$ \\
\hline Never been married & $150(46.6)$ \\
\hline Divorced, widowed, or separated & $15(4.7)$ \\
\hline \multicolumn{2}{|l|}{ Educational level } \\
\hline High school diploma or less & $44(13.7)$ \\
\hline Some college & $110(34.2)$ \\
\hline College graduate & $139(43.2)$ \\
\hline Advanced degree & $27(8.4)$ \\
\hline \multicolumn{2}{|l|}{ Employment status } \\
\hline Employed for wages/self-employed & $255(79.2)$ \\
\hline Student & $17(5.3)$ \\
\hline Retired & $6(1.9)$ \\
\hline Out-of-work or unable to work & $15(4.7)$ \\
\hline Homemaker & $27(8.4)$ \\
\hline \multicolumn{2}{|l|}{ Household income } \\
\hline Less than $\$ 25,000$ & $78(24.2)$ \\
\hline$\$ 25,001$ to $\$ 50,000$ & $109(33.9)$ \\
\hline$\$ 50,001$ to $\$ 75,000$ & $64(19.9)$ \\
\hline More than $\$ 75,000$ & $64(19.9)$ \\
\hline Don’t want to say & $5(1.6)$ \\
\hline
\end{tabular}

\section{Procedure}

The study was created on the Qualtrics survey platform and administered through Amazon Mechanical Turk. Participants were randomly assigned to one of six conditions: 
(1) news narrative on depression (text only; control), (2) news narrative on depression with photos, (3) news narrative on depression with cartoons, (4) news narrative on bipolar disorder (text only; control), (5) news narrative on bipolar disorder with photos, and (6) news narrative on bipolar disorder with cartoons. Before viewing the story in their assigned condition, participants were asked about their familiarity with mental illness (Holmes et al., 1999) and their preference for visual versus verbal information processing (Childers, Houston, Heckler, 1985). After participants answered these questions, they viewed the story. In order to ensure that participants paid attention to the story, a timer was inserted into the survey platform so that participants had to spend a minimum amount of time reading the story and would not be able to advance through the study until they had met or exceeded that minimum time limit. This timer was set as 2 minutes for the text-only conditions and 3 minutes and 30 seconds for the text-plus-image conditions.

After viewing the story, participants were asked open- and closed-ended questions that evaluated, for example, their emotional and cognitive response to the story; their attitudes, behavioral intentions, and behavior toward people with mental illness; their response to the images and text in the story; and demographic characteristics (for example, age, gender, political ideology).

The time required for completing the study was estimated to be about 20 minutes; however, participants were given an hour to complete the study. Study completion time ranged from 6 to 50 minutes. The mean study completion time was 15.95 minutes +/6.82 minutes. Most participants took 11.35 minutes to complete the study. 


\section{Stimuli}

The experimental stimuli comprised six types of news stories - three on depression and three on bipolar disorder. Of the three stories on depression, one had only text and served as a control; one had photos in addition to text; and one had cartoons in addition to text. The three stories on bipolar disorder were also of the same type. Participants were randomly assigned to view one of these six types of stories.

Text. The content of the news narratives on depression and bipolar disorder were based on a variety of sources: published news stories on these illnesses from news organizations such as NPR, The Daily Herald, the BBC; university news outlets such as the University of Missouri's Vox Magazine and the University of Kansas's University Daily Kansan; and non-profit organizations such as psychcentral.com and pendulum.org. The stories on both depression and bipolar disorder told the story of central character Eliza Smith's experience with depression and bipolar disorder, respectively. Each story described the onset of her illness, the symptoms, diagnosis, and treatment. The stories were both about 800 to 1000 words long. Both stories were formatted to look like online news stories.

Images. As explained above, for both the depression and bipolar condition, one story had photos in addition to text and one had cartoons in addition to text.

Photographs. An undergraduate female student at the University of Missouri served as the model for Eliza Smith, the protagonist in both the depression and bipolar disorder narratives. A master's student at the University of Missouri and the author served as models for other female story characters. Both the undergraduate and master's student models signed a "model release form," and in doing so, provided written consent 
for the photographs to be presented at research conferences and published in academic journals or other academic literature. Please see Appendix B for an example of a model release form. Also, both students were compensated $\$ 20$ for their time.

Almost all photographs were taken over a two-week period on the campus of the University of Missouri in February 2016. The photographer is a doctoral journalism student and photojournalist at the University of Missouri who volunteered to work on this project. The depression story had a total of 12 photographs; the bipolar story, 10. All photographs were in black and white. For the depression story, one of the photographs was an image of an MRI scan showing differences in activity levels between a brain with and without depression. This image is not original—it was sourced from Google Images, was converted to black and white, and was altered so that it was text-free.

Cartoons. The cartoons in the study were operationalized as follows: similar to the photographs but simplistic, devoid of detail, and depicting the inner emotions/thoughts of the story characters. The cartoons for the cartoon versions of the depression and bipolar stories were based on the photographs in the corresponding photo versions of the stories. This was to ensure that the images were as similar as possible. The cartoons were designed by a graphic designer, who is a graduate of the journalism master's program at the University of Missouri.

The photographs were converted to simple black and white cartoon illustrations, such that the cartoon was identical to its corresponding photo with regard to size and placement of people and objects. This was achieved by drawing over the photographs using Adobe Illustrator. The photographs were then removed, leaving behind the outlines of the images. Cartoon elements, such as visual metaphors, personified emotions, and 
thought bubbles, were then added in. The author provided ideas for these elements for each cartoon to the designer. The ideas for these elements were inspired by graphic novels about mental illness (for example, "Marbles: Mania, Depression, Michelangelo, and Me: A Graphic Memoir" (Forney, 2012); "Living with a Black Dog: His Name is Depression" (Johnstone, 2006); and "Mom's Cancer" (Fies, 2006). Some ideas also emerged from brainstorming discussions with the graphic designer. The 22 cartoons were designed over a five-month period, ranging from February to June 2016. The designer was compensated $\$ 326.50$ for her work.

Two journalism/communication faculty members in midwestern universities and two doctoral journalism/communication students (one of whom is also a trained mentalhealth therapist) from midwestern and northeastern universities were requested to read the stories and provide feedback on the text and images. Edits/revisions were made based on their feedback.

\section{Pre-Test}

To ensure that the cartoons were similar to their corresponding photographs from which they were derived, a sample of 330 U.S. MTurk adults with a HIT approval rate of $95 \%$ or above and 100 or more HITs approved were recruited to take an "assessing image similarity" study. An MTurk sample was used to ensure that the participants who assessed the similarity of the images used in the stimuli and those who took the main study had a similar demographic makeup. Informed consent was obtained from all participants. Participants were given 15 minutes to complete the study (estimated study duration time, 2 minutes). Participants were compensated $\$ 0.30$ for their time.

Participants were randomly assigned to one of 22 conditions. (There were 22 
conditions because there were a total of 22 pairs of images. A sample size of 330 ensured that at least 15 participants were assigned to each condition.) Each condition had a total of two pairs of images. Each pair was made up of one cartoon and one photograph. It is important to note that the order in which the images were presented — cartoon first and then photograph or photograph first and then cartoon — was varied to ensure that there were no order effects.

In each condition, participants were presented with the first pair of images, asked to compare the images, and then asked to indicate (on a scale of 1 to $5 ; 1$, strongly disagree; 5 , strongly agree) how much they agreed or disagreed with the following nine statements that assessed image similarity: "The photograph and cartoon visually resemble each other"; "The photograph and cartoon are of the same size"; "The cartoon and photograph depict the same event"; "The placement of people in both images is the same"; "The placement of objects in both images is the same"; "The cartoon is a simplified, less detailed version of the photograph"; "The cartoon shows what the person is feeling or thinking"; "The cartoon shows what the person is feeling or thinking"; "The photograph shows what the person is feeling or thinking"; "The photograph is more realistic/lifelike than the cartoon.”

After participants had finished rating the first pair of images, they were presented with the second pair of images, asked to compare them, and then asked to indicate (on a scale of 1 to $5 ; 1$, strongly disagree; 5 , strongly agree) how much they agreed or disagreed with the same nine statements.

The mean study completion time was 3.6 minutes ( $\mathrm{SD}=+/-14.34$ seconds). Most participants took 2.6 minutes to complete the study. Most participants agreed that the 
images were visually similar $(\mathrm{M}=3.99, \mathrm{SD}=.41)$, of the same size $(\mathrm{M}=4.36, \mathrm{SD}=.37)$, depicted the same event $(\mathrm{M}=4.02, \mathrm{SD}=.43)$, and that the placement of people in both images was similar $(\mathrm{M}=4.14, \mathrm{SD}=.52)$. Further, most participants agreed that the cartoons ( $\mathrm{M}=4.0, \mathrm{SD}=.62)$ showed what the person was thinking/feeling whereas the photographs did not $(\mathrm{M}=2.54, \mathrm{SD}=.56)$. This difference was significant $(\mathrm{t}(86)=11.51$, $\mathrm{p}<0.01)$

\section{Dependent Variables}

Message credibility. Based on Appelman and Sundar (2015), message credibility was measured by asking participants to rate (1, strongly disagree; 7 , strongly agree) how well the following adjectives described the content they just read: "accurate, authentic, and reliable." Reliability was high (Cronbach's alpha for DText $=.83$; Cronbach's alpha for DPhoto $=.85 ;$ Cronbach's alpha for DCartoon $=.87 ;$ Cronbach's alpha for BPhoto $=$ .91; Cronbach's alpha for BText $=.89$; Cronbach's alpha for BCartoon $=.89)$. So the scores for the three items for the depression and bipolar conditions each were summed to create a single scale (DTextCredibility: $\mathrm{M}=17.34, \mathrm{SD}=2.91$; DPhotoCredibility: 18.63 , $\mathrm{SD}=2.56$; DCartoonCredibility: $\mathrm{M}=18.56, \mathrm{SD}=2.15$; BPhotoCredibility: $\mathrm{M}=18.33$, $\mathrm{SD}=2.24$; BTextCredibility: $\mathrm{M}=18.42, \mathrm{SD}=2.88$; BCartoonCredibility: $\mathrm{M}=18.1, \mathrm{SD}$ $=2.36$ ). Higher values (up to 21 possible) indicate more credibility.

Identification. Drawing on Tal-Or and Cohen (2010), identification was measured by asking participants to rate the following items (1, strongly disagree; 7 , strongly agree): "I understand the events in the story the way Eliza understood them", "I think I understand Eliza well," "While reading the story, I felt like Eliza felt," "While reading the story," "I felt like I could really get inside Eliza's head," "I tend to understand 
why Eliza did what she did." Reliability was high (Cronbach's alpha for DTextIdentification $=.90$, Cronbach's alpha for DPhotoIdentification $=.87$, Cronbach's alpha for DCartoonIdentification $=.87$, Cronbach's alpha for BPhoto $=.87$, Cronbach's alpha for BText $=.79$, Cronbach's alpha for BCartoon $=.77$ ). So the scores for the five items for the depression and bipolar conditions each were summed to create a single scale (DTextIdentification: $\mathrm{M}=24.02, \mathrm{SD}=7.1$; DPhotoIdentification: $\mathrm{M}=27.58, \mathrm{SD}=5.06$; DCartoondentification: $\mathrm{M}=26.27, \mathrm{SD}=5.31$; BPhotoIdentification: $\mathrm{M}=24.6, \mathrm{SD}=$ 5.73, BTextIdentification: $\mathrm{M}=25.56, \mathrm{SD}=4.54, \mathrm{BCartoondentification:} \mathrm{M}=25.12, \mathrm{SD}$ $=4.84$ ). Higher values (up to 35 possible) indicate stronger identification.

Prejudicial feelings. Drawing on Ramasubramanian (2010), participants' prejudicial feelings were assessed by asking them to what extent the following adjectives described their feelings toward the story's protagonist: "discomfort, nervousness, disgust, dislike, fear, and anger" (1, strongly disagree; 7 , strongly agree). Reliability was high (Cronbach's alpha for DTextPrejudicialFeelings $=.94$, Cronbach's alpha for DPhotoPrejudicialFeelings $=.88$, Cronbach's alpha for DCartoonPrejudicialFeelings $=.91$, BPhotoPrejudicialFeelings $=.92$, BTextPrejudicialFeelings $=.91$, BCartoonPrejudicialFeelings $=.90$ ). So the scores for the six items for the depression and bipolar conditions each were summed to create a single scale (DTextPrejudicialFeelings: $\mathrm{M}=11.85, \mathrm{SD}=7.48$, DPhotoPrejudicialFeelings: $\mathrm{M}=10.08, \mathrm{SD}=5.88$, DCartoonPrejudicialFeelings: $\mathrm{M}=10.75, \mathrm{SD}=6.78$, BPhotoPrejudicialFeelings: $\mathrm{M}=$ 10.67, $\mathrm{SD}=6.02$, BTextPrejudicialFeelings: $\mathrm{M}=10.13, \mathrm{SD}=5.64$, BCartoonPrejudicialFeelings: $\mathrm{M}=10.69, \mathrm{SD}=6.37$ ). Higher values (up to 42 possible) indicate stronger prejudicial feelings. 
Pity. Drawing on Oliver et al. (2013), participants were asked to what extent the following adjectives described their feelings toward the story's protagonist: pity, “sympathy, sorry for, worried for" (1, strongly disagree; 7, strongly agree). Reliability was high (Cronbach's alpha for DTextPity $=.89$, Cronbach's alpha for DPhotoPity $=.74$, Cronbach's alpha for DCartoonPity $=.78$, Cronbach's alpha for BPhotoPity $=.83$, Cronbach's alpha for BTextPity $=.75$, Cronbach's alpha for BCartoontPity $=.73$ ). So the scores for the four items for pity for the depression and bipolar conditions each were summed to create a single scale (DTextPity: $\mathrm{M}=18.44, \mathrm{SD}=6.28$; DPhotoPity: $\mathrm{M}=$ 20.77, $\mathrm{SD}=4.32$; DCartoonPity: $\mathrm{M}=20.04, \mathrm{SD}=4.59$; BPhotoPity: $\mathrm{M}=18.83, \mathrm{SD}=$ 5.27 ; BTextPity: $\mathrm{M}=18.71, \mathrm{SD}=4.47$; BCartoonPity: $\mathrm{M}=20.22, \mathrm{SD}=4.34$ ). Higher values (up to 28 possible) indicate stronger feelings of pity.

Connectedness. Drawing on Oliver et al. (2013), participants were asked to what extent the following adjectives described their feelings toward the story's protagonist: “closeness, caring, connection, compassion” (1, strongly disagree; 7, strongly agree). Reliability was high (Cronbach's alpha for DTextConnectedness $=.89$, Cronbach's alpha for DPhotoConnectedness $=.86$, Cronbach's alpha for DCartoonConnectedness $=.83$, Cronbach's alpha for BPhotoConnectedness $=.89$, Cronbach's alpha for BTextConnectedness $=.79$, Cronbach's alpha for BCartoonConnectedness $=.78$ ). So the scores for the four items for connectedness for the depression and bipolar conditions each were summed to create a single scale (DTextConnectedness: $\mathrm{M}=18.43, \mathrm{SD}=6.18$, DPhotoConnectedness: $\mathrm{M}=22.64, \mathrm{SD}=4.31$, DCartoonConnectedness: $\mathrm{M}=19.85, \mathrm{SD}$ =4.5; BPhotoConnectedness: $\mathrm{M}=19.65, \mathrm{SD}=5.25$; BTextConnectedness: $\mathrm{M}=19.76$, $\mathrm{SD}=4.38$; BCartoonConnectedness: $\mathrm{M}=20.5, \mathrm{SD}=4.31$ ). Higher values (up to 28 
possible) indicate stronger feelings of connectedness.

Perceived severity. Drawing on Chang (2013), participants were asked to rate the following items: "Depression is a serious disease"; "Depression has serious consequences" (1, strongly disagree; 7 , strongly agree). For the bipolar disorder condition, the same items were used except that "depression" was replaced with "bipolar disorder." Reliability was high (Cronbach's alpha for DTextPerceivedSeverity $=.88$, Cronbach's alpha for DPhotoPerceivedSeverity $=.82$, Cronbach's alpha for DCartoonPerceivedSeverity $=.70$, Cronbach's alpha for BPhotoPerceivedSeverity $=.93$, Cronbach's alpha for BTextPerceivedSeverity $=.91$, Cronbach's alpha for BCartoonPerceivedSeverity $=.91$ ). So the scores for the two items for perceived severity for the depression and bipolar conditions each were summed to create a single scale (BTextPerceivedSeverity: $\mathrm{M}=12.64, \mathrm{SD}=1.75$, BPhotoPerceivedSeverity: $\mathrm{M}=12.38$, $\mathrm{SD}=1.72$, BCartoonPerceivedSeverity: $\mathrm{M}=12.67, \mathrm{SD}=1.58$ ). Higher values (up to 14 ) indicate stronger perceived severity.

Perceived response efficacy. Drawing on Chang (2013), participants were asked to rate the following items: "The story provides effective strategies to treat depression" "The story provides effective recommendations to treat depression"; "The story provides useful information to treat depression" (1, strongly disagree; 7 , strongly agree). For the bipolar disorder condition, the same items were used except that "depression" was replaced with "bipolar disorder." Reliability was high (Cronbach's alpha for DTextPerceivedResponseEfficacy $=.89$, Cronbach's alpha for DPhotoPerceivedResponseEfficacy $=.90$. Cronbach's alpha for DCartoonPerceivedResponseEfficacy $=.96$, Cronbach's alpha for 
BPhotoPerceivedResponseEfficacy $=.89$, Cronbach's alpha for

BTextPerceivedResponseEfficacy $=.90$, Cronbach's alpha for

BCartoonPerceivedResponseEfficacy $=.90$ ). So the scores for the three items for

perceived response efficacy for the depression and bipolar conditions each were summed

to create a single scale (DTextPerceivedResponseEfficacy: $\mathrm{M}=16.00, \mathrm{SD}=2.97$,

DPhotoPerceivedResponseEfficacy: $\mathrm{M}=16.57, \mathrm{SD}=3.18$,

DCartoonPerceivedResponseEfficacy: $\mathrm{M}=16.58, \mathrm{SD}=3.32$;

BPhotoPerceivedResponseEfficacy: $\mathrm{M}=15.33, \mathrm{SD}=3.11$;

BTextPerceivedResponseEfficacy: $\mathrm{M}=15.18, \mathrm{SD}=3.83$;

BCartoonPerceivedResponseEfficacy: $\mathrm{M}=15.37, \mathrm{SD}=3.54$ ). Higher values (up to 14 )

indicate stronger perceived response efficacy.

Personal responsibility beliefs. Drawing on Corrigan et al. (2003), participants'

personal responsibility beliefs regarding mental illness were measured by asking them to rate the following items: "I think people with depression have themselves to blame for their illness (1, strongly disagree; 7, strongly agree);" "Do you think the cause of depression is under personal control? (1, not at all under personal control; 7, completely under personal control);" "How responsible do you think are people with depression for their illness? (1, not at all responsible; 7, very responsible)." For the bipolar disorder condition, the same items were used except that "depression" was replaced with "bipolar disorder." Reliability was high (Cronbach's alpha for DTextPerceivedResponsibility = .75 , Cronbach's alpha for DPhotoPerceivedResponsibility $=.82$, Cronbach's alpha for DCartoonPerceivedResponsibility $=.83$, Cronbach's alpha for BPhotoPerceivedResponsibility $=.89$, Cronbach's alpha for 
BTextPerceivedResponsibility $=.67$, Cronbach's alpha for

BCartoonPerceivedResponsibility $=.81$ ) So the scores for the three items for perceived response efficacy for the depression and bipolar conditions each were summed to create a single scale (DTextPerceivedResponsibility: $\mathrm{M}=8.06, \mathrm{SD}=3.62$,

DPhotoPerceivedResponsibility: $\mathrm{M}=6.74, \mathrm{SD}=3.61$, DCartoonPerceivedResponsibility: $\mathrm{M}=7.6, \mathrm{SD}=3.81$; BPhotoPerceivedResponsibility: $\mathrm{M}=5.46, \mathrm{SD}=3.75$; BCartoonPerceivedResponsibility: $\mathrm{M}=5.1, \mathrm{SD}=3.13$ ). Higher values (up to 21) indicate stronger perceived responsibility beliefs.

Social distance. Drawing on Link et al. (1987), social distancing responses toward people with mental illness was assessed by asking participants to rate the following items: "If you were a landlord, would you rent out an apartment to someone with depression?" "Would you be willing to accept a person with depression as your coworker?" "Would you be willing to accept a person with depression as your neighbor?" "Would you be willing to hire a person with depression for taking care of your kids for a few hours?" "Would you be willing to accept a person with depression as your in-law?" "Would you introduce someone with depression to your friends?" "If a friend of yours was looking for an employee, would you recommend someone with depression? (1, strongly unwilling; 7, strongly willing)." For the bipolar disorder condition, the same items were used except that "depression" was replaced with "bipolar disorder."

Reliability was high (Cronbach's alpha for DTextSocialDistance $=.92$, Cronbach's alpha for DPhotoSocialDistance $=.89$, Cronbach's alpha for DCartoonSocialDistance $=.89$, Cronbach's alpha for BPhotoSocialDistance $=.90$, Cronbach's alpha for BTextSocialDistance $=.88$, Cronbach's alpha for BCartoonSocialDistance $=.90$ ). So the 
scores for the seven items for social distance for the depression and bipolar conditions each were summed to create a single scale (DTextSocialDistance: $\mathrm{M}=36.94, \mathrm{SD}=9.02$, DPhotoSocialDistance: $\mathrm{M}=38.89, \mathrm{SD}=7.61$, DCartoonSocialDistance: $\mathrm{M}=39.29, \mathrm{SD}$ $=6.80$, BPhotoSocialDistance: $\mathrm{M}=38.33, \mathrm{SD}=7.57$; BTextSocialDistance: $\mathrm{M}=37.64$, $\mathrm{SD}=7.31 ;$ BCartoonSocialDistance: $\mathrm{M}=37.57, \mathrm{SD}=8.57$ ). Higher values (up to 49) indicate less likelihood to have social distance behavioral intentions.

Coercion-segregation. Drawing on Corrigan et al. (2003), coercion-segregation responses toward the story's protagonist was assessed by asking participants to rate the following items: "I think Eliza poses a risk to her neighbors unless she is hospitalized," "I think it would be best for Eliza's community if she were put away in a psychiatric hospital," "How much do you think a psychiatric institution, where Eliza can be kept away from her neighbors is best?" "If I were in charge of Eliza's treatment, I would force her to live in a group home (1, strongly disagree; 7, strongly agree)." For the bipolar disorder condition, the same items were used except that "depression" was replaced with “bipolar disorder.” Reliability was high (Cronbach's alpha for DTextCoercionSegregation $=.95$, Cronbach's alpha for DPhotoCoercion-Segregation $=.96$, Cronbach's alpha for DCartoonCoercion-Segregation= .98 , Cronbach's alpha for BPhotoCoercionSegregation $=.96$, Cronbach's alpha for BTextCoercion-Segregation= .94 , Cronbach's alpha for BCartoonCoercion-Segregation= .94). So the scores for the four items for coercion-segregation for the depression and bipolar conditions each were summed to create a single scale (DTextCoercion-Segregation: $\mathrm{M}=7.13, \mathrm{SD}=4.96$, DPhotoCoercion-Segregation: $\mathrm{M}=7.25, \mathrm{SD}=5.66$, DCartoonCoercion-Segregation: $\mathrm{M}$ $=6.35, \mathrm{SD}=4.24 ;$ BPhotoCoercion-Segregation: $\mathrm{M}=6.38, \mathrm{SD}=4.06$; BTextCoercion- 
Segregation: $\mathrm{M}=6.22, \mathrm{SD}=4.27$; $\mathrm{BCartoonCoercion-Segregation:} \mathrm{M}=6.02, \mathrm{SD}=$ 3.91). Higher values (up to 28) indicate stronger coercion-segregation behavioral intentions.

Mental-health policy support. Drawing on Barry et al. (2014), policy support in regard to mental illness was assessed by asking participants to rate the following items: "Do you favor or oppose requiring insurance companies to offer benefits for the treatment of mental illness that are equivalent to benefits for other medical services?" "Do you favor or oppose increasing government spending on the treatment of mental illness?" "Do you favor or oppose increasing government spending on programs to subsidize housing costs for people with mental illness?" "Do you favor or oppose increasing government spending on programs that help people with drug addiction/mental illness find jobs and provide on-the-job support as needed?" (1, strongly oppose; 7 , strongly favor). For the bipolar disorder condition, the same items were used except that "depression" was replaced with "bipolar disorder." Reliability was high (Cronbach's alpha for DTextMental-IllnessPolicySupport $=.88$, Cronbach's alpha for DPhotoMentalIllnessPolicySupport $=.83$, Cronbach's alpha for DCartoonMental-IllnessPolicySupport $=.85$, BPhotoMental-IllnessPolicySupport $=.89, \mathrm{BTextMental-IllnessPolicySupport}=$ .89 , BCartoonMental-IllnessPolicySupport $=.83$ ). So the scores for the four items for the depression and bipolar conditions each were summed to create a single scale (DTextMental-IllnessPolicySupport: $\mathrm{M}=21.35, \mathrm{SD}=4.88$; DPhotoMentalIllnessPolicySupport: $\mathrm{M}=23.38, \mathrm{SD}=4.2$; DCartoonMental-IllnessPolicySupport: $\mathrm{M}=$ 22.73, $\mathrm{SD}=4.58$; BPhotoMental-IllnessPolicySupport: $\mathrm{M}=23.4, \mathrm{SD}=3.81$; BTextMental-IllnessPolicySupport: $\mathrm{M}=22.71, \mathrm{SD}=5.16$; BCartoonMental- 
IllnessPolicySupport: $\mathrm{M}=24.39, \mathrm{SD}=3.99$ ). Higher values (up to 28 ) indicate stronger policy support behavioral intentions.

Dissemination likelihood. Drawing on Smith (2012), participants' likelihood of sharing the message was assessed by asking participants the following question: "How likely would you be to share this message with: family, close friends, colleagues/classmates/coworkers" (1, not likely; 5, very likely; 6, not applicable). Reliability was high (Cronbach's alpha for DTextDisseminationLikelihood $=.96$, Cronbach's alpha for DPhotoDisseminationLikelihood $=.92$, Cronbach's alpha for DCartoonDisseminationLikelihood $=.95$, Cronbach's alpha for BPhotoDisseminationLikelihood $=.90$, Cronbach's alpha for BTextDisseminationLikelihood $=.87$, Cronbach's alpha for BCartoonDisseminationLikelihood $=.90)$. So the scores for the three items for the depression and bipolar conditions each were summed to create a single scale (DTextDisseminationLikelihood: $\mathrm{M}=9.07, \mathrm{SD}=4.15$; DPhotoDisseminationLikelihood: $\mathrm{M}=11.25, \mathrm{SD}=3.46$; DCartoonDisseminationLikelihood: $\mathrm{M}=10.29, \mathrm{SD}=4.14$; BPhotoDisseminationLikelihood: $\mathrm{M}=10.19, \mathrm{SD}=3.66$; BCartoonDisseminationLikelihood: $\mathrm{M}=9.86, \mathrm{SD}=4.08$; BTextDisseminationLikelihood: $\mathrm{M}=10.24, \mathrm{SD}=3.52$ ). Higher values (up to 15 ) indicate stronger likelihood of disseminating the message.

Donation. As a behavioral measure, participants were asked the following questions: "Are you willing to donate some portion of your MTurk earnings from this study to fund mental health services?" (1, yes; 2 , no) "If yes, how much (range, 0.1 to 2 dollars)." Regardless of how they responded to this question, no money was actually 
deducted from the amount participants' were compensated (that is, \$2) for participation in the study-All participants were paid $\$ 2$.

Recall. To assess how well participants' remembered the story, they were asked the following two questions in the depression condition: (1) "Eliza Smith had all of the following symptoms except for: Episodes of mania, lack of concentration, feeling of exhaustion, difficulty interacting with people." (2) "Eliza Smith did all of the following to improve her mental health except for: Taking a semester off, medication, cooking classes, talk therapy." For the bipolar condition, participants were asked the following two questions: (1) "Which of the following best describes how the story explained the states experienced by a person with bipolar disorder? "Each person may experience a variety of states, ranging from mania to depression. Treatment triggers mania. Mania and depression do not occur simultaneously. Depression gradually develops into mania during the course of a lifetime." (2) "What was said about students' access to treatment? Students have relatively easy access to treatment at their universities. Students don't need much treatment because the illness is at the early stages of development. Students have a better chance of getting help because of their flexible schedules. Community mentalhealth care can be expensive."

Manipulation check. In the text version of both the depression and bipolar conditions, participants were asked the following question: Did the story you just read have images in addition to text (yes/no)? In the photo and cartoon versions of both the depression and bipolar conditions, participants were asked the following questions: "Did the story you just read have images in addition to text (yes/no)?" "If yes, what type of images were they (photographs/cartoons)?" The manipulation was successful. For the 
depression story, in the text condition, $94.44 \%$ of participants answered the question "Did the story you just read have images in addition to text (yes/no)?” correctly. In the cartoon condition, all participants answered the question correctly. Further, of these, $98.2 \%$ correctly identified the story images as cartoons in response to the question "If yes, what type of images were they (photographs/cartoons)?” In the photo condition, 98.11\% correctly indicated that the story had images in addition to text, and of these participants, all correctly identified the images as photographs in response to the question "If yes, what type of images were they (photographs/cartoons)?"

For the bipolar story, in both the photo and cartoon conditions, all participants correctly answered the question "Did the story you just read have images in addition to text (yes/no)?" correctly. Further, all correctly identified the images as either photos or cartoons in response to the question "If yes, what type of images were they (photographs/cartoons)?"' In the text condition, $90.9 \%$ correctly indicated that the story had no images.

Open-ended questions. In the image versions of both the depression and bipolar disorder stories, just before the demographic questions, participants were asked the following two questions: "Please list at least 3 thoughts about the text in the story you just read." "Please list at least 3 thoughts about the images in the story you just read." For participants who received the text version of the stories, they were asked the following question: "Please list at least 3 thoughts about the story you just read."

\section{Moderator Variables}

Level of contact with mental illness. Drawing on Holmes et al. (1999), before participants were exposed to the stimulus, their familiarity with mental illness was 
assessed by asking them to read items that described situations involving different degrees of contact with people with mental illness. Participants were asked to check all situations they had experienced in their lifetime. Each item was assigned a rank-order score, and the index for familiarity with mental illness was the rank score of the situation that described the closest degree of contact with persons with mental illness. The following are the items along with the rank order of the item: "I have watched a movie or television show in which a character depicted a person with mental illness" (3). "My job involves providing services/treatment for persons with a mental illness" (8). "I have observed, in passing, a person I believe may have had a mental illness" (2). "I have observed persons with a mental illness on a frequent basis" (5). "I have a mental illness" (12). "I have worked with a person who had a mental illness at my place of employment" (6). "I have never observed a person that I was aware had a mental illness" (1). "My job includes providing services to persons with a mental illness" (7). "A friend of the family has a mental illness" (9). "I have a relative who has a mental illness" (10). "I have watched a documentary about mental illness" (4). "I live with a person who has a mental illness" (11). "I do not have any of the listed experiences with mental illness" (0).

Style of processing. Before exposure to the stimulus, to determine participants' preference for visual or verbal processing, they were asked to rate the following items ( 1 , always true; 4, always false): "I enjoy doing work that requires the use of words." "There are some special times in my life that I like to relive by mentally "picturing" just how everything looked." "I can never seem to find the right word when I need it." "I do a lot of reading." "When I'm trying to learn something new, I'd rather watch a demonstration than read how to do it." "I think I often use words in the wrong way." "I enjoy learning 
new words." "I like to picture how I could fix up my apartment or a room if I could buy anything I wanted." "I often make written notes to myself." "I like to daydream." "I generally prefer to use a diagram rather than a written set of instructions." "I like to doodle." "I find it helps to think in terms of mental pictures when doing many things." "After I meet someone for the first time, I can usually remember what they look like but not much about them." "I like to think of synonyms for words." "When I have forgotten something, I frequently try to form a mental picture to remember it." "I like learning new words." "I prefer to read instructions about how to do something rather than have someone show me." "I prefer activities that require a lot of reading." "I seldom daydream." "I spend very little time trying to increase my vocabulary." "My thinking often consists of mental "pictures" or images." Reliability was low: Cronbach's alpha was .69. Therefore, this variable was not considered for further data analysis. Consequently, hypotheses H31a and H32a could not be tested.

Liberal ideology. At the end of the study, participants were asked demographic questions, which included the questions "How conservative are you?" and "How liberal are you?" The variable "How conservative are you" was recoded, and then values were compared with the variable "How liberal are you." Data from 22 subjects who did not have similar values on both items was deleted. Then, the two variables were summed to form a 2-item measure of degree of liberal ideology (Cronbach's alpha $=.95 ; \mathrm{M}=7.14$, $\mathrm{SD}=2.44$ ). Higher values (up to 10 ) indicate stronger liberal ideology.

\section{Data Analysis}

Quantitative data were analyzed in SPSS22 using one-way analysis of variance (ANOVA) and the independent samples t-test. Further, the PROCESS macro (Hayes 
2013) was used to conduct moderation and mediation analyses. Data were screened for univariate outliers $(n=19)$ by deleting data points for each variable that were greater than +/- 3.29 standard deviations away from the mean. Qualitative data (responses to the openended questions) were coded using the constant-comparative method (Glaser \& Strauss, 1967). 


\section{Chapter 4: Results}

\section{Message Credibility}

For participants who viewed the story on depression, there was a significant difference between the three groups (photo, cartoon, and text (control)) with regard to perceptions of message credibility such that participants who saw the photo version $(\mathrm{M}=$ 18.63) or the cartoon version (18.56) reported more credibility than those who saw the text-only version $(\mathrm{M}=17.34)(\mathrm{F}(2,159)=5.25, \mathrm{p}=.006)$. Thus, H1 was supported. Tukey's post-hoc analyses revealed significant differences in perceived credibility between the text and photo $(\mathrm{p}=.013)$ and text and cartoon narratives $(\mathrm{p}=.018)$. However, there was no significant difference in perceived credibility between the photo and cartoon narratives $(\mathrm{p}=.99)(\mathrm{RQ} 1)$.

For participants who viewed the story on bipolar disorder, there was no significant differences between the three groups $(\mathrm{M}$ (photo) $=18.27, \mathrm{M}$ (text) $=18.70, \mathrm{M}$ $($ cartoon $)=18.1)$ with regard to perceptions of message credibility $(\mathrm{F}(2,155)=1.068, \mathrm{p}=$ .346). Thus, H2 was not supported. There was also no significant difference in perceived credibility between the photo and cartoon narratives $(\mathrm{t}(100)=.39, \mathrm{p}=.70)(\mathrm{RQ} 2)$.

\section{Perceived Severity and Perceived Response Efficacy}

For participants who viewed the story on depression, there were no significant differences among the three groups with regard to perceived disease severity (M (photo) $=12.64, \mathrm{M}($ text $)=12.60, \mathrm{M}($ cartoon $)=12.64)(\mathrm{F}(2,160)=.008, \mathrm{p}=.992)$. Thus, $\mathrm{H} 3$ and H3a were not supported. Further, there was also no significant difference among these groups with regard to perceived response efficacy $(\mathrm{M}($ photo $)=16.57, \mathrm{M}($ text $)=$ 
16.00, $\mathrm{M}($ cartoon $)=16.58(\mathrm{~F}(2,161)=.595, \mathrm{p}=.553)$. Thus, H5 and H5a were not supported.

For participants who viewed the story on bipolar disorder, there were no significant differences among the three groups with regard to perceived disease severity $(\mathrm{M}($ photo $)=12.38, \mathrm{M}($ text $)=12.91, \mathrm{M}($ cartoon $)=12.67)(\mathrm{F}(2,155)=1.634, \mathrm{p}=.199)$ Thus, H4 and H4a were not supported. There were also no significant differences among these three groups with regard to perceived response efficacy $(\mathrm{M}$ (photo) $=15.33, \mathrm{M}$ $($ text $)=15.18, \mathrm{M}($ cartoon $)=15.37)(\mathrm{F}(2,157)=.043, \mathrm{p}=.958)$. Thus, H6 and H6a were not supported.

\section{Identification}

For participants who viewed the story on depression, there was a significant difference among the three groups (photo, cartoon, and text (control)) with regard to identification, such that participants who saw the photo version $(\mathrm{M}=27.58)$ reported more identification with the story character than those who saw the cartoon $(\mathrm{M}=26.27)$ or text-only version $(\mathrm{M}=24.36)(\mathrm{F}(2,160)=4.25, \mathrm{p}=.017)$. Thus, H7 was supported.

For participants who viewed the story on bipolar disorder, however, there were no significant differences between the three groups $(\mathrm{M}$ (photo $)=24.6, \mathrm{M}($ text $)=25.56, \mathrm{M}$ $($ cartoon $)=25.12)(\mathrm{F}(2,157)=.49, \mathrm{p}=.61)$ with regard to identification. Thus, H8 was not supported.

\section{Affective Reactions}

Connectedness. For participants who viewed the story on depression, there was a significant difference among the three groups (photo, cartoon, and text (control)) with regard to identification, such that participants who saw the photo version $(M=22.64)$ 
reported more connectedness with the story character than those who saw the cartoon (M $=19.85)$ or text-only version $(\mathrm{M}=18.43)(\mathrm{F}(2,160)=9.474, \mathrm{p}<.01)$. Thus, $\mathrm{H} 9$ was supported. For participants who viewed the story on bipolar disorder, there was no significant difference among the three groups $(\mathrm{M}($ photo $)=19.65, \mathrm{M}($ text $)=19.76, \mathrm{M}$ $($ cartoon $)=20.5)$ with regard to connectedness $(\mathrm{F}(2,157)=.491, \mathrm{p}=.613)$. Thus, H10 was not supported.

Pity. For participants who viewed the story on depression, there was a marginally significant difference with regard to pity toward the story's protagonist among the three groups with photo eliciting the most pity, following by cartoon and then text (M (photo) $=20.77, \mathrm{M}($ text $)=18.44, \mathrm{M}($ cartoon $)=20.04)(\mathrm{F}(2,161)=2.85, \mathrm{p}=.059)$; however, Levene's test for homogeneity of variance was significant $(\mathrm{p}=.02)$. Thus, H11 was not supported. For participants who viewed the story on bipolar disorder, there was no significant difference with regard to pity toward the story's protagonist among the three groups $(\mathrm{M}($ photo $)=18.83, \mathrm{M}($ text $)=18.71, \mathrm{M}($ cartoon $)=20.22)(\mathrm{F}(2,157)=1.643, \mathrm{p}$ $=.197)$. Thus, H12 was not supported.

Prejudicial feelings. For participants who viewed the story on depression, there were no significant differences in prejudicial feelings toward the story's protagonist among the three groups $(\mathrm{M}($ photo $)=10.08, \mathrm{M}($ text $)=11.85, \mathrm{M}($ cartoon $)=10.3)(\mathrm{F}(2$, $160)=1.2, p=.304)$. Thus, H13 was not supported. For participants who viewed the story on bipolar disorder, there were no significant differences in prejudicial feelings toward the story's protagonist among the three groups $(\mathrm{M}$ (photo) $=10.67, \mathrm{M}($ text $)=$ $10.13, \mathrm{M}($ cartoon $)=10.69)(\mathrm{F}(2,157)=.152, \mathrm{p}=.859)$. Thus, $\mathrm{H} 14$ was not supported. 


\section{Perceived Responsibility Beliefs}

For participants who viewed the story on depression, there were no significant differences among the three groups with regard to perceived responsibility beliefs ( $\mathrm{M}$ $($ photo $)=6.74, \mathrm{M}($ text $)=8.06, \mathrm{M}($ cartoon $)=7.6)(\mathrm{F}(2,160)=1.765, \mathrm{p}=.175)$. Thus H15 was not supported. For those who viewed the story on bipolar disorder, there was no significant difference between those exposed to the photo $(\mathrm{M}=4.9, \mathrm{SD}=2.5)$ and cartoon $(\mathrm{M}=5.1, \mathrm{SD}=3.13)$ versions of the story $(\mathrm{t}(1,95.012)=.726)$. Thus, H16 was not supported. (Those exposed to the text version of the story were not considered for this analysis because the perceived responsibility scale for that group was found to be not reliable (Cronbach's alpha $<.70)$ ).

\section{Behavioral Intentions and Behavior}

Mental health policy support. For participants who viewed the story on depression, there was no significant difference among the groups with regard to support for mental-health policy $(\mathrm{M}($ photo $)=23.38, \mathrm{M}($ text $)=21.62, \mathrm{M}($ cartoon $)=22.73)(\mathrm{F}$ $(2,160)=2.123, \mathrm{p}=.123)$. Thus, H17 was not supported. For participants who viewed the story on bipolar disorder, there was no significant difference among the groups with regard to support for mental-health policy $(\mathrm{M}($ photo $)=23.40, \mathrm{M}($ text $)=23.06, \mathrm{M}$ $($ cartoon $)=24.39)(\mathrm{F}(2,156)=1.473, \mathrm{p}=.232)$. Thus, H18 was not supported.

Social distance. For participants who viewed the story on depression, there was no significant difference among the groups with regard to social distance $(\mathrm{M}$ (photo) $=$ $39.50, \mathrm{M}($ text $)=37.42, \mathrm{M}($ cartoon $)=39.8)(\mathrm{F}(2,158)=1.888, \mathrm{p}=.155)$. Thus, H19 was not supported. For participants who viewed the story on bipolar disorder, there was also no significant difference among the groups with regard to social distance (M (photo) 
$=38.33, \mathrm{M}($ text $)=37.64, \mathrm{M}($ cartoon $)=37.57)(\mathrm{F}(2,157)=.15, \mathrm{p}=.861)$. Thus, $\mathrm{H} 20$ was not supported.

Dissemination likelihood. For participants who viewed the story on depression, there was a significant difference between the groups with regard to message dissemination likelihood, such that those in the photo condition were $(\mathrm{M}($ photo $)=11.15)$ more likely to disseminate the message compared to those in the text $(\mathrm{M}($ text $)=9.07)$ or cartoon conditions $(\mathrm{M}($ cartoon $)=9.82)(\mathrm{F}(2,161)=3.813, \mathrm{p}=.024)$. Thus, H21 was supported. For participants who viewed the story on bipolar disorder, there was no significant difference between the groups with regard to message dissemination likelihood $(\mathrm{M}($ photo $)=9.79, \mathrm{M}($ text $)=9.96, \mathrm{M}($ cartoon $)=9.86)(\mathrm{F}(2,156)=.029, \mathrm{p}=$ .971). Thus, H22 was not supported.

Coercion-segregation. For participants who viewed the story on depression, there was no significant difference among the groups with regard to coercion-segregation $(\mathrm{M}($ photo $)=6.53, \mathrm{M}($ text $)=6.81, \mathrm{M}($ cartoon $)=6.35)(\mathrm{F}(2,158)=.156, \mathrm{p}=.855)$. Thus, H23 was not supported. For participants who viewed the story on bipolar disorder, there was no significant difference among the groups with regard to coercion-segregation $(\mathrm{M}($ photo $)=6.38, \mathrm{M}($ text $)=5.91, \mathrm{M}($ cartoon $)=5.70)(\mathrm{F}(2,155)=.474, \mathrm{p}=.623)$. Thus H24 was not supported.

Behavior. For participants who viewed the story on depression, there was no significant difference between the three groups with regard to donation behavior $\left(\mathrm{c}^{2}(2, \mathrm{~N}\right.$ $=162)=4.07, \mathrm{p}=.694)$ ). There was a marginally significant difference between the cartoon and photo conditions, however, such that those in the cartoon condition were more likely to donate than those in the photo condition $\left.\left(\mathrm{c}^{2}(2, \mathrm{~N}=108)=3.8, \mathrm{p}=.051\right)\right)$. 
Thus, H25 was partially supported. For participants who viewed the story on bipolar disorder, there was no significant difference among the groups with regard to donation behavior $\left.\left(\mathrm{c}^{2}(2, \mathrm{~N}=157)=.86, \mathrm{p}=.651\right)\right)$. Thus, $\mathrm{H} 26$ was not supported.

Recall. For participants who viewed the story on depression, there was no significant difference among the groups with regard to recall question $1\left(\mathrm{c}^{2}(6, \mathrm{~N}=162)\right.$ $=3.87, \mathrm{p}=.131))$. Thus, $\mathrm{H} 31$ was not supported. For participants who viewed the story on bipolar disorder, there was no significant difference among the groups with regard to the two recall questions (recall question $1: \mathrm{c}^{2}(4, \mathrm{~N}=158)=3.12, \mathrm{p}=.539$; recall question 2: $\left.\mathrm{c}^{2}(4, \mathrm{~N}=157)=4.89, \mathrm{p}=.299\right)$. Thus, $\mathrm{H} 32$ was not supported.

\section{Interaction Effects With the Moderator Familiarity with Mental Illness}

Identification. For participants who viewed the depression story or the story on bipolar disorder, there was no significant interaction between familiarity with mental illness and image type on identification ( $p>$.05). Thus, H7a and H8a were not supported.

Connectedness. For participants who viewed the depression story or the story on bipolar disorder, there was no interaction between familiarity with mental illness and image type on connectedness $(\mathrm{p}>.05)$. Thus, H9a and H10a were not supported.

Pity. For participants who viewed the depression story or the story on bipolar disorder, there was no interaction between familiarity with mental illness and image type on pity ( $\mathrm{p}>.05)$. Thus, H11a and H12a were not supported.

Prejudicial feelings. For participants who viewed the depression story or the story on bipolar disorder, there was no interaction between familiarity with mental illness and image type on prejudicial feelings ( $p>.05$ ). Thus, H13a and H14a were not supported. 
Perceived responsibility. For participants who viewed the depression story or the story on bipolar disorder, there was no interaction between familiarity with mental illness and image type on perceived responsibility $(p>.05)$. Thus, H15a and H16a were not supported.

Mental-health policy support. For participants who viewed the depression story or the story on bipolar disorder, there was no interaction between familiarity with mental illness and image type on mental health policy support ( $p>.05)$. Thus, H17c and H18c were not supported.

Social distancing behavioral intention. For participants who viewed the depression story or the story on bipolar disorder, there was no interaction between familiarity with mental illness and image type on social distancing behavioral intentions $(\mathrm{p}>.05)$. Thus, H19c and H20c were not supported.

Dissemination likelihood. For participants who viewed the depression story or the story on bipolar disorder, there was a marginally significant interaction between familiarity with mental illness and image type (text vs. photo) on dissemination likelihood $(\mathrm{B}=.43, \mathrm{SE}=.22, \mathrm{t}=1.97, \mathrm{p}=.05)$. Thus, $\mathrm{H} 21 \mathrm{c}$ was partially supported. Specifically, for participants with moderate and high familiarity with mental illness, those exposed to the photo condition $(\mathrm{M}=11.15)$ were more likely to disseminate the story compared to those in the cartoon condition $(\mathrm{M}=9.07)$ (familiarity with mental illness at the mean: $\mathrm{B}=2.22, \mathrm{SE}=.72, \mathrm{t}=3.08, \mathrm{p}<.01,95 \% \mathrm{CI}=.79-3.66$; familiarity with mental illness $1 \mathrm{SD}$ above the mean: $\mathrm{B}=3.67, \mathrm{SE}=1.02, \mathrm{t}=3.59, \mathrm{p}<.01,95 \% \mathrm{CI}=1.64$ - 5.70) (Figure 2). For those with low familiarity with mental illness, exposure to the photo version versus the text version did not produce a difference in dissemination 
likelihood $(\mathrm{p}=.46)$. For participants who viewed the story on bipolar disorder, there was no interaction between familiarity with mental illness and image type on dissemination likelihood ( $\mathrm{p}>.05)$. Thus, H22c was not supported.

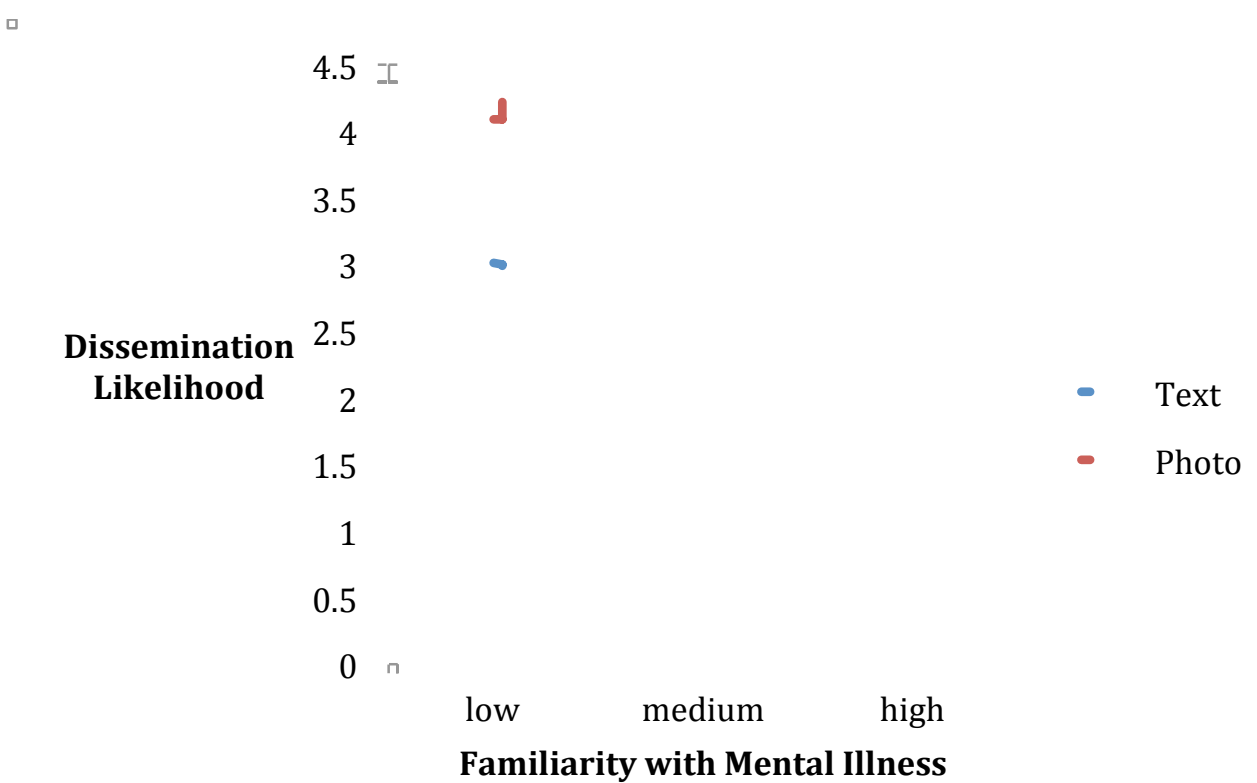

Figure 2: Moderation interaction between image type (text/photo) and familiarity with mental illness on dissemination likelihood.

Coercion-segregation behavioral intention. For participants who viewed the depression story or the story on bipolar disorder, there was no interaction between familiarity with mental illness and image type on coercion-segregation behavioral intentions ( $\mathrm{p}>.05)$. Thus, $\mathrm{H} 23 \mathrm{c}$ and $\mathrm{H} 24 \mathrm{c}$ were not supported.

Behavior. For participants who viewed the depression story or the story on bipolar disorder, there was no interaction between familiarity with mental illness and image type on donation behavior ( $\mathrm{p}>.05$ ). Thus, $\mathrm{H} 25 \mathrm{c}$ and $\mathrm{H} 26 \mathrm{c}$ were not supported.

\section{Interaction Effects With the Moderator Political Ideology}

Mental-health policy support. For participants who viewed the depression story or the story on bipolar disorder, there was no interaction between liberal ideology and 
image type on support for mental-health policy $(\mathrm{p}>.05)$. Thus, H27 and H29 were not supported.

Coercion-segregation. For participants who viewed the depression story or the story on bipolar disorder, there was no interaction between liberal ideology and image type on coercion-segregation behavioral intention $(\mathrm{p}>.05)$. Thus, H28 and H30 were not supported.

\section{Interaction Effects With the Mediator Identification}

Mental-health policy support. For those who viewed the story on depression, image type did influence mental-health policy support indirectly through the mediator identification (Image type: Text vs Photo: $\mathrm{ab}=.2583,95 \%$ bootstrap CI $=.0726$ to .5425 ; effect size $=.12$ ). Participants in the photo condition were significantly more likely to identify with the story protagonist than those in the text condition, which in turn led to greater support mental health policy; however, there was no significant difference between the photo and cartoon conditions (Figure 3). Thus, H17a was supported. For those who viewed the story on bipolar disorder, image type did not significantly influence mental-health policy support through the mediator identification $(\mathrm{p}>.05)$. Thus, H18a was not supported. 


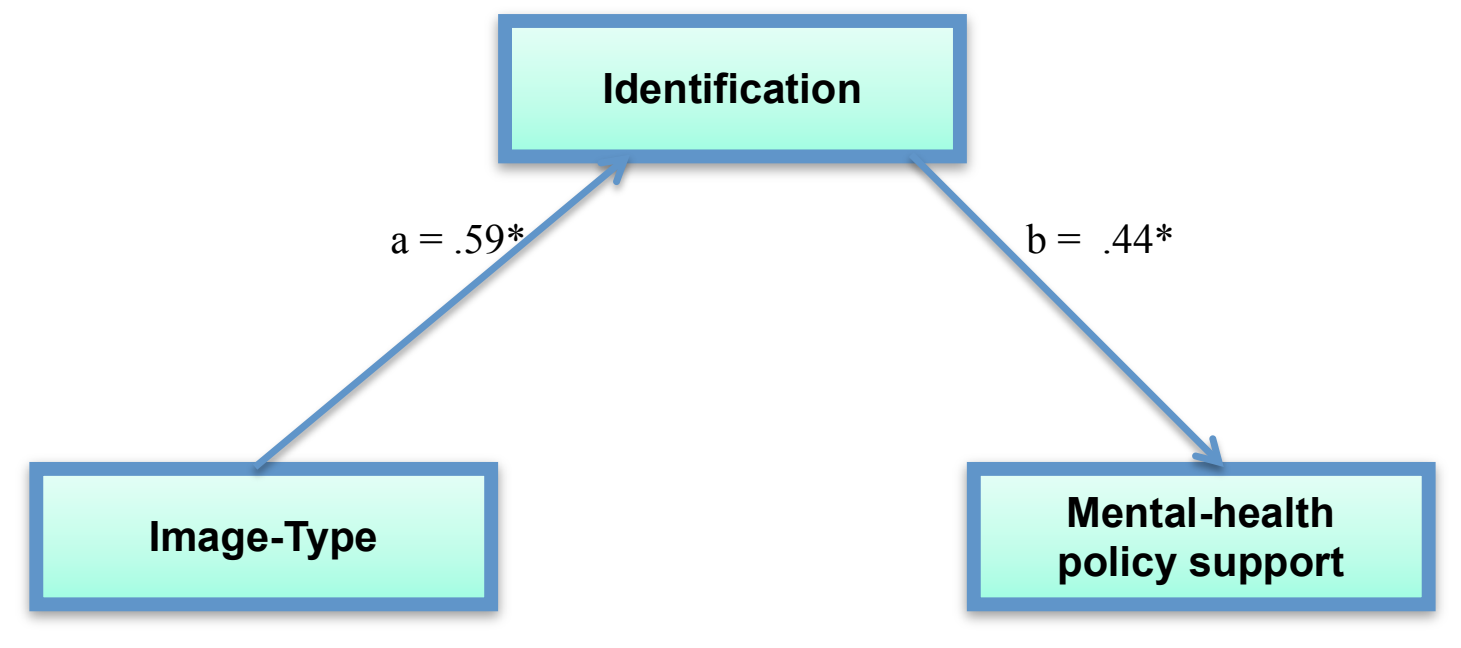

Figure 3. Mediation of the relationship between image type (text/photo) and mental health policy support by identification.

Social distance behavioral intention. For those who viewed the story on depression, image type did not influence social distance behavioral intentions through the mediator identification ( $\mathrm{p}>.05)$. Thus, H19a and H20a were not supported.

Coercion-segregation. For those who viewed the story on depression, image type did not influence coercion-segregation behavioral intentions indirectly through the mediator identification ( $\mathrm{p}>.05)$. Thus, H23a and H24a were not supported.

Dissemination likelihood. For those who viewed the story on depression, image type did influence dissemination likelihood indirectly through the mediator identification (Image type: Text vs Photo: $\mathrm{ab}=.3131,95 \%$ bootstrap $\mathrm{CI}=.1031$ to .6192 ; effect size $=$ .12). Participants in the photo condition were significantly more likely to identify with the story protagonist than those in the text condition, which in turn led to higher likelihood of sharing the message; however, there was no significant difference between the photo and cartoon conditions (Figure 4). Thus, H21a was supported. For those who viewed the story on bipolar disorder, image type did not significantly influence dissemination likelihood through the mediator identification $(p>.05)$. Thus, H22a was 
not supported.

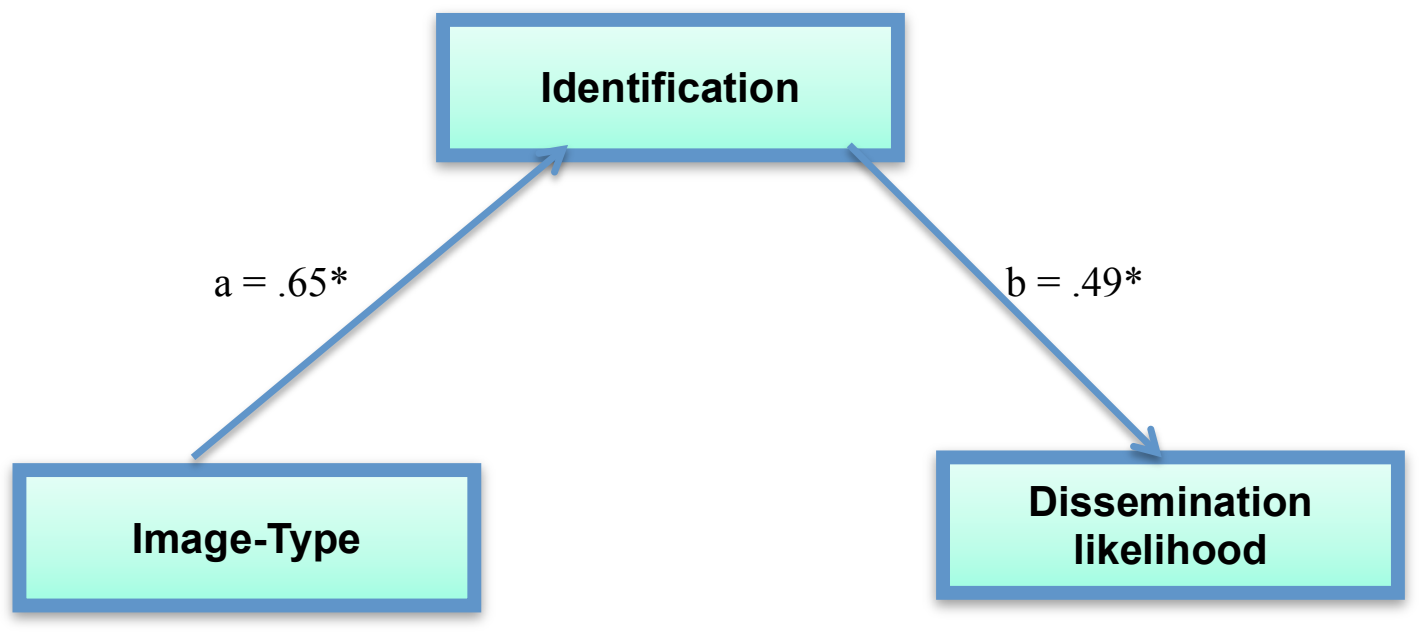

Figure 4. Mediation of the relationship between image type (text/photo) and dissemination likelihood by identification.

Behavior. For participants who viewed the depression story or the story on bipolar disorder, there was no interaction between image type and identification on behavior $(\mathrm{p}>.05)$. Thus, H25a and H26a were not supported.

\section{Interaction Effects With the Mediator Connectedness}

Social distance. For those who viewed the story on depression, image type did influence social distance behavioral intentions indirectly through the mediator connectedness (Image type: Text vs Photo: $\mathrm{ab}=.2133,95 \%$ bootstrap CI $=.0540$ to .2780 ; effect size $=.10$ ). Those in the photo condition were more likely to connect more with the story protagonist than those in the text condition, which in turn led to less social distancing behavioral intentions; however, there was no significant difference between the photo and cartoon condition (Figure 5). Thus, H19b was supported. For those who viewed the story on bipolar disorder, image type did not influence mental social distancing behavioral intention through the mediator connectedness $(\mathrm{p}>.05)$. Thus, H20b 
was not supported.

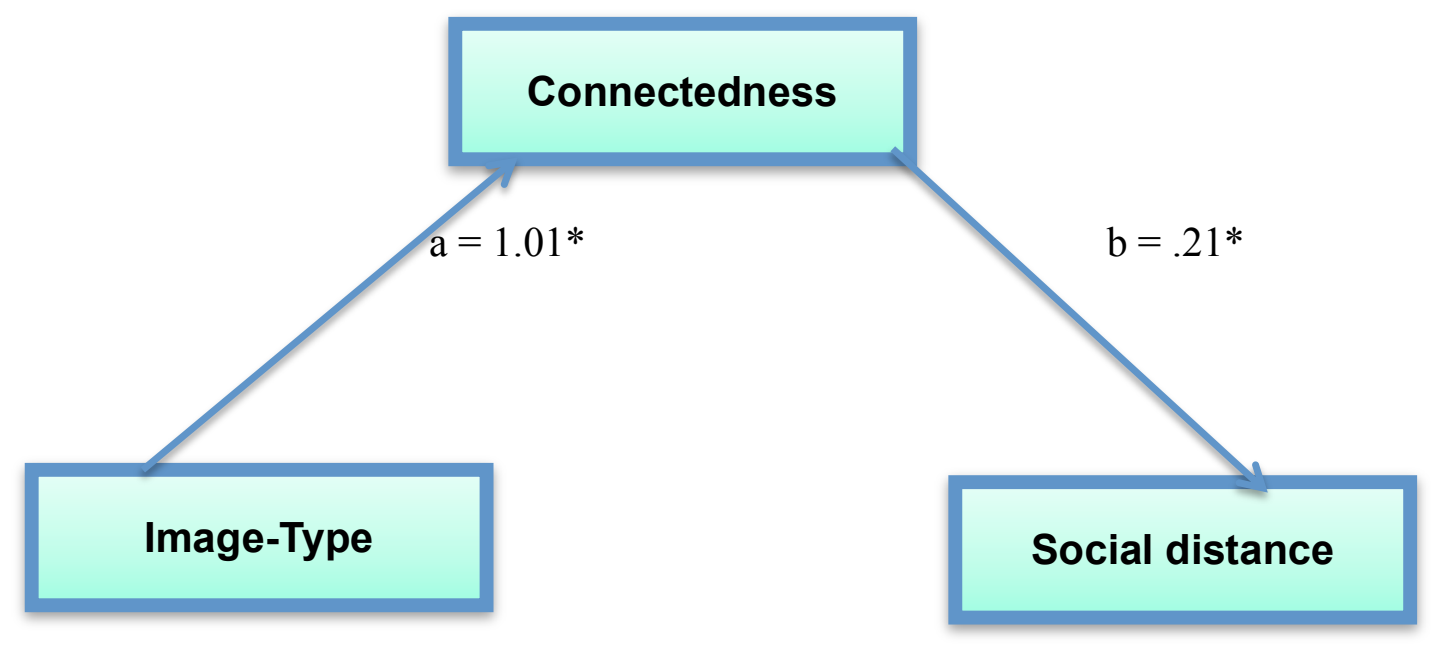

Figure 5: Mediation of the relationship between image type (text/photo) and social distance by connectedness.

Mental-health policy support. For those who viewed the story on depression, image type did influence mental-health policy support indirectly through the mediator connectedness (Image type: Text vs Photo: $\mathrm{ab}=.3234,95 \%$ bootstrap $\mathrm{CI}=.1356$ to .6135 ; effect size $=.14)$. Participants in the photo condition were more likely to connect more with the story protagonist than those in the text condition, which in turn led to more support for mental health policy (Figure 6); however, there was no significant difference between the photo and cartoon condition. Thus, H17b was partially supported. For those who viewed the story on bipolar disorder, image type did not influence mental health policy support through the mediator connectedness $(\mathrm{p}>.05)$. Thus, H18b was not supported. 


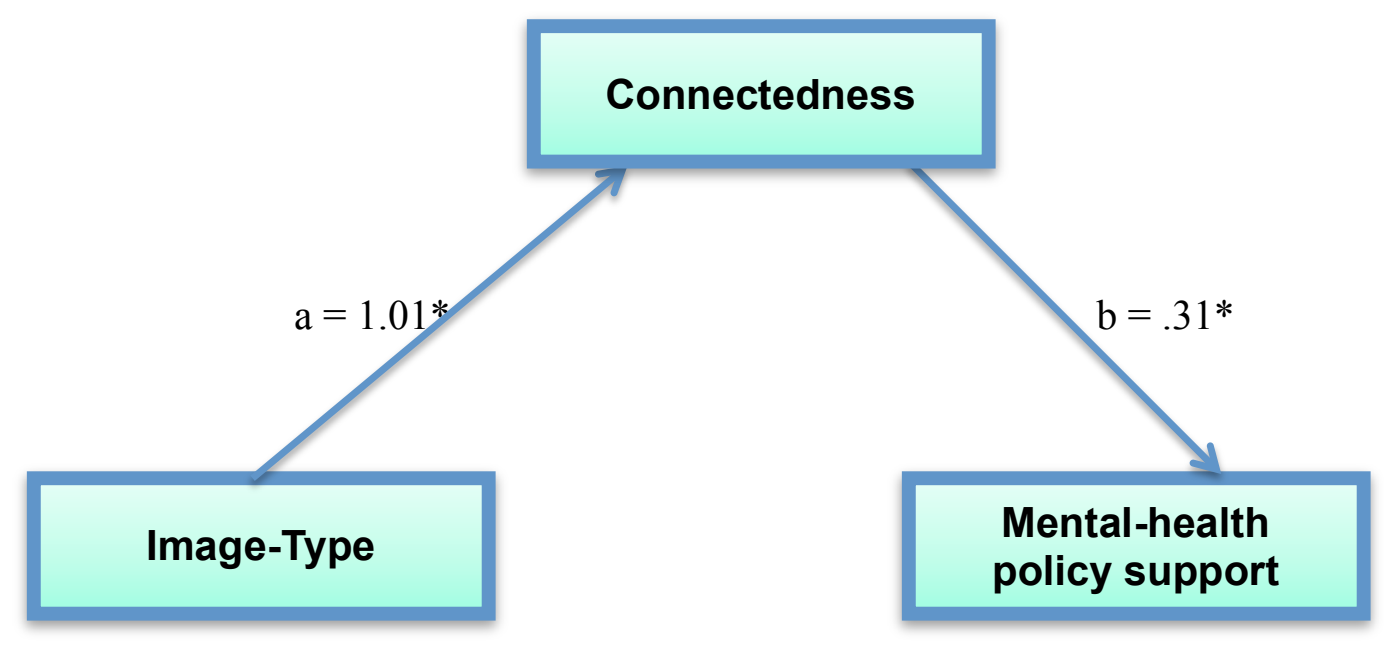

Figure 6: Mediation of the relationship between image type (text/photo) and mental health policy support by connectedness.

Coercion-segregation. For those who viewed the story on depression, image type did not influence coercion segregation behavioral intentions indirectly through the mediator connectedness $(\mathrm{p}>.05)$. Thus, $\mathrm{H} 23 \mathrm{~b}$ and $\mathrm{H} 24 \mathrm{~b}$ were not supported.

Dissemination likelihood. For those who viewed the story on depression, image type did influence message dissemination likelihood indirectly through the mediator connectedness (Image type: Text vs Photo: $\mathrm{ab}=.4443,95 \%$ bootstrap CI $=.2288$ to .7660 ; effect size $=.17$ ). Participants in the photo condition were more likely to connect more with the story protagonist than those in the text condition, which in turn led to more likelihood to share the message (Figure 7); however, there was no significant difference between the photo and cartoon condition. Thus, H21b was supported. For those who viewed the story on bipolar disorder, image type did not influence dissemination likelihood through the mediator connectedness $(\mathrm{p}>.05)$. Thus, H22b was not supported. 


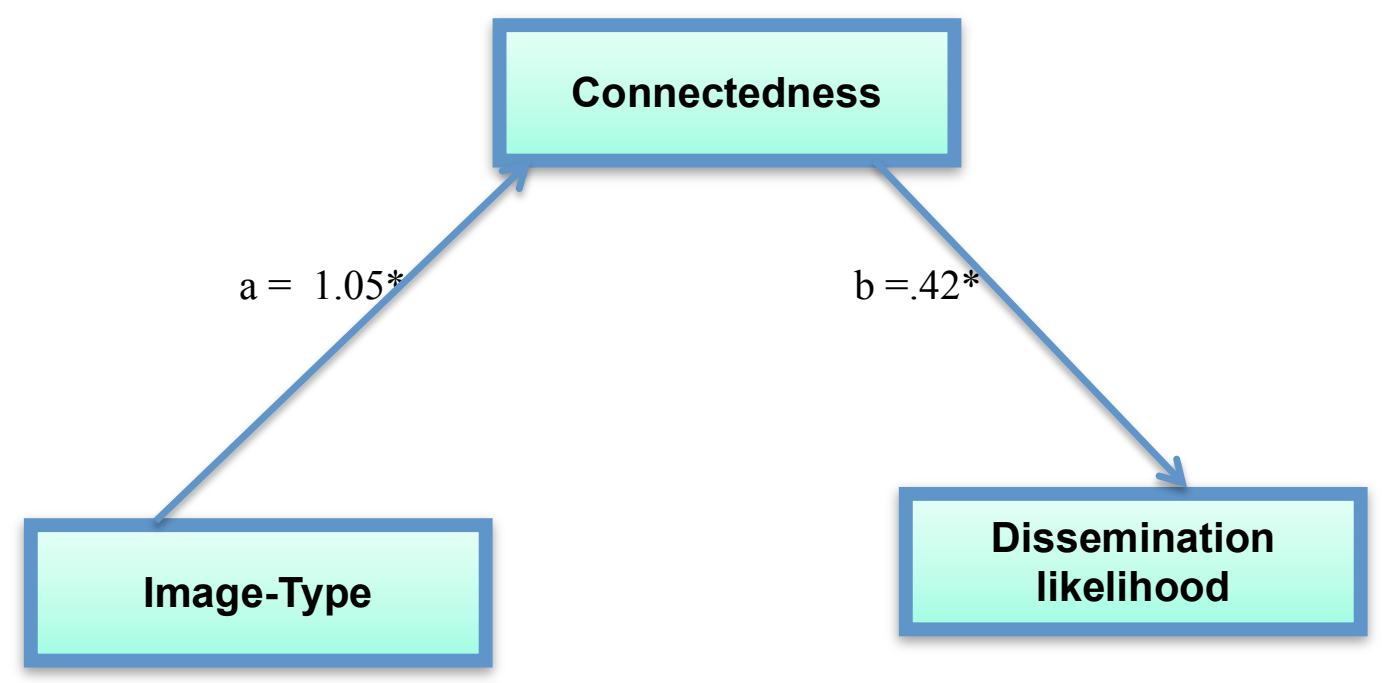

Figure 7: Mediation of the relationship between image type (text/photo) and dissemination likelihood by connectedness.

Behavior. For participants who viewed the depression story or the story on bipolar disorder, there was no interaction between image type and connectedness on behavior $(\mathrm{p}>.05)$. Thus, $\mathrm{H} 25 \mathrm{~b}$ and $\mathrm{H} 26 \mathrm{~b}$ were not supported.

\section{Analysis of Open-ended Responses}

In the photo and cartoon conditions, at the end of the survey, participants were asked to list at least three thoughts about (1) the images in the story and (2) the text in the story. In the text-only versions of the stories, participants were asked to list at least three thoughts about the story they had just read. Participants' responses ranged from a few words to a few sentences. The open-ended responses were compared and analyzed for themes.

Depression condition. Dominant themes in the open-ended responses of the depression condition are described below.

Text. Most participants in the text-alone condition reported a positive response to 
the story. A couple of respondents described the story as "unrealistic" and "stereotypical" and questioned whether the protagonist actually had depression and whether the neurological factors were definitely implicated in the disease. Some of the comments also indicated that the text was engaging enough for participants' to be transported into the narrative, for example, "I really found myself rooting for Eliza to get the help she needed."

Photographs. In the depression condition, some participants questioned the authenticity of the photographs, describing them as "posed," "forced," "staged," and "unrealistic." However, most reported that they liked the photos in the story. Positive comments included that the photos were well-taken, were authentic, complemented the text well, helped sustain attention and interest, and most importantly, put a human face on the issue.

Humanizing. Several participants noted that the photographs helped humanize the issue. The photos helped them visualize the protagonist's struggles, and in doing so, helped them "relate," feel "empathy" and "closeness," and made it easier to understand the issue. One participant wrote: "I imagined myself being in her shoes while looking at the photographs." Another said:

One of the image[s] of Eliza laying down is touching because she fell [feels] useless. Image of Eliza at her University bring joy because she willing ot [to] learn in the face of depression. The last image of her cooking is great because it show that she can overcome it.

Some participants also noted that the protagonist looked "normal" and like "a regular college student." One said that this aspect of the photos helped convey the invisibility of depression: "Eliza seemed like someone who would be popular with a lot of friends showing that you can seem like a perfectly normal person and suffer from crippling 
anxiety and depression."

Cartoons. Most participants who viewed the story on depression with cartoon images had a positive response to the images and said that the cartoons made the story interesting/complemented the story and aided in comprehension. Some, however, felt that the images were "comical" and detracted from the seriousness of the issue.

Illuminating/Illustrative. Several participants said that the images were simple and easy to understand. They did a good job of portraying the symptoms of depression as well as treatment strategies. By showing how the protagonist was feeling, they helped "visualize" the disease. A participant commented on how the "black and white" nature of the artwork showed "the black and white thinking of depression." Another said:

The dark image by her as depression was accurate to the portrayal of dark energy felt from depression. The hard time concentrating at the desk looked true to life. The people's feet around her being so much larger than her reminded me of feelings about feeling small around people.

Another felt that the images helped lighten the mood of the story: "They made the story seem light hearted and easier to read, they also made it seem less real in a way but it made it easier to read than with photos of a person persay[per se]."

Inappropriate. Some participants had a negative response to the cartoon images, describing the images as "childish," "comical," and "dull." A few noted that the images took away from the somber nature of the issue. One participant said:

The images seemed inappropriate given the seriousness of the issue. They seemed to trivialize the matter. They weren't informative nor particularly evocative. The only possibly benefit is that by trivializing mental illness the images might remove some of the fear and stigma related to such diseases.

Bipolar disorder condition. In the bipolar versions of the stories, for the photo condition, most participants had a positive response to the images. Participants described 
the protagonist as "pretty," "normal," and "happy." (Participants in the depression photo condition also commented on how "pretty" and "normal" the protagonist looked, but the frequency of these comments was higher in the bipolar photo condition.) Although some said that they identified or related with the story, it was in the open-ended responses for the cartoon condition of the bipolar story that the word "humanizing" came up several times. One participant wrote:

At first I thought they seemed a bit out of place given how serious the subject matter. As I continued reading the article, though, I felt that they added a sort of humanizing aspect to the story \& made it feel more accessible/less dreary [not that I personally found it dreary, but I can see how some might]. In general, I thought they did a great job at depicting the overarching issues presented in the text in a way that was both succinct and interesting.

Several described the cartoons as providing information or being informative.

One participant wrote: "You could almost just use the cartoons and leave out the story and still get a good idea of the situation."

Overall, in both the depression and bipolar conditions, the qualitative, open-ended responses reflected the quantitative findings. The open-ended responses also help make sense of the quantitative findings. It appears that in the depression condition, the photographs evoked an emotional response, whereas in the bipolar condition, the cartoons were more effective at doing so. 


\section{Chapter 5: Discussion}

Comics have long been used in health communication messages, such as health campaigns, because they are believed to offer many advantages in communicating health issues (McNicol, 2014). Further, comics journalism, news about current events in comics format (Williams, 2005; CJR review, p. 2), is an emerging genre that is believed to offer the advantage of combing "literal retelling" and "artistic symbolism" to tell compelling stories Williams, 2005; CJR review, p. 6). However, whether the purported emotional and cognitive benefits of comics are true is not clear because there is not much empirical research on the persuasive effects of comics on readers' cognition, affect, and behavior. Inspired by graphic novels, this study investigated whether news narratives on mental illness (one on depression and the other on bipolar disorder) accompanied by real images, specifically, photographs, would be more persuasive in terms of reducing mental illness stigma than news narratives accompanied by cartoons (the building block of comics) based on those photographs or news narratives alone (that is, without any images).

Results indicate that for participants who viewed the three different types of stories on depression (text, text + photo, or text + cartoon), images (photo/cartoon) had a greater persuasive effect than text alone. Participants in the photo condition reported higher identification and connectedness with the story's protagonist than those in the text or cartoon conditions. Further, those in the photo or cartoon condition reported higher perceived credibility than those in the text condition. Photos also led to higher support for mental-health policy (than text alone) and less social distance behavioral intention (than text alone). Also, photos led to greater likelihood to disseminate the news story (than text 
alone), especially for those with greater familiarity with mental illness. Open-ended responses indicated that participants found the photographs "humanizing" whereas the cartoons were perceived to serve an explanatory purpose. For participants who viewed the three different types of stories on bipolar disorder (text, text + photo, or text + cartoon), the results were non-significant and inconclusive: Neither photo nor text nor cartoon exerted a dominant pattern of persuasive effects.

\section{Interpretation of Results}

Depression condition. Participants who saw the photo or cartoon version of the reported significantly higher perceived credibility than those who saw the text-only version of the story. Because news photos "convey a concrete believability that words do not" (Lowrey, 1998, as cited in Greer and Gosen, 2002, p. 5), it is understandable that those in the photo condition reported more perceived credibility. Further, Taylor (2000) argues that photographs are accepted as authentic not because they provide "absolute or even substantial proof" (p. 131) but because of convention. He says that readers have come to accept/believe that "photographs used to authenticate accounts are themselves the products of an eyewitness and that they record actual, historical moments" (p. 131). This partly reflects the findings in the study in that it was not just photos but also cartoons that elicited higher perceived credibility responses. This indicates that the images, whether representational/realistic (photographs) or interpretational/artistic (cartoons) lent a concreteness to the story, which in turn made the story more authentic.

However, it's interesting that those in the cartoon condition also reported more perceived credibility than those in the text condition. The cartoons did not reduce the credibility of the story. In fact, those in the photo condition reported only slightly higher 
credibility scores $(M=18.63)$ than those in the cartoon condition $(M=18.56)$. It appears that the cartoons were not perceived as interpretive artistic renderings but as objective, true accounts of the protagonist's experience with mental illness. This is noteworthy given that cartoons are "frequently and unreflectively been associated with the stigmatized categories of cognitive immaturity, impairment, and disability" (Squier, 2008, p. 130), which could lead to low credibility perceptions. It's possible that the participants in this study did not make such associations because they were familiar with the comics journalism genre and so understood that the information in the story was not fabricated (Williams, 1005; Nyberg, 2006). The absence of significant differences in credibility perceptions between real and artistic/fictitious images was also found in another science communication study, which examined the effects of different types of images placed above a news story about neuroscience (Gruber and Dickerson, 2012).

With regard to identification, participants who saw the photo version $(M=27.58)$ reported significantly more identification with the story character than those who saw the text-only version $(\mathrm{M}=24.36)$. This might because several photos in the depression story showed the protagonist's suffering and could be defined as agonistic images, that is, images that trigger "agonistic vigilance" (Zillman, 1998) — the need to screen one's surroundings for danger. Images of people suffering or those that depict the aftermath of destruction fall under this category (Zillman, Knobloch, \& Yu, 2001). Research has show that people spend more time looking at photographs of individuals who were victimized (Zillman, Knobloch, \& Yu, 2009). Several photographs in the depression condition depicted the protagonist grappling with her disease. Photographs portrayed her symptoms 
of sadness, hopelessness, and loneliness. It appears that like in previous studies, such victim portrayals drew attention and interest.

Identification for the cartoon condition $(\mathrm{M}=26.27)$ was not significantly lower than that for the photo condition $(\mathrm{M}=27.58)$. This might be because as comics scholars postulate, the simplistic and abstract nature of images in cartoons and the elimination of unnecessary details help readers project themselves onto cartoon characters and identify more closely with them (Green \& Myers, 2010; McCloud, 1993). Also, according to comics scholars, comics artists use realistic pictures to portray the outside world and cartoons to portray the world within (McCloud, 1993; p. 41). However, in this study, even though the cartoons portrayed the world within the character, that is, the protagonist's symptoms of depression, there was probably not much difference between the photo and cartoon conditions because the symptoms of depression are perceptible.

Similar to identification, photos also elicited highest levels of connectedness among the three groups. There was no significant difference between the three groups with regard to prejudicial feelings. However, prejudicial feelings were lowest for the photo condition.

There were no significant differences among the three groups with regard to perceived disease severity or response efficacy. In both cases, the means for photo $(\mathrm{M}=$ 12.64 and 15.33 , respectively) and cartoon $(M=12.64$ and 15.37 , respectively) were higher and almost identical than the mean for text $(\mathrm{M}=12.60$ and 15.18). Given that the participants in the sample reported having high familiarity with mental illness, this outcome is not surprising as participants probably had high literacy with regard to depression and so the addition of images did not increase risk perception because of a 
ceiling effect.

The higher means for the image conditions with regard to perceived severity is in line with previous research that has shown that information in pictorial format increases risk perceptions (Lee, Cameron, Wunsche, \& Stevens, 2011), helps the reader imagine the warning signs of the health issue being discussed (Keller \& Block, 1997) and increases recall of the negative consequences of the health threat (Boer, Huurne, \& Taal, 2006). One explanation for this effect is that the salience and vividness of images makes pictorial information more accessible, thereby increasing risk perceptions (Zillman \& Brosius, 2000). Further, text with pictures has been found to activate more mental imagery than text without pictures (Miller \& Stoica, 2003; Walters, Sparks, \& Herington, 2007), which in turn has the potential to increase perceptions of disease severity (Chang, 2013).

Higher values for the image conditions with regard to perceived response efficacy is also in line with previous research that suggests that observing how others behave helps to evaluate the pros and cons of the recommended behavior and offers a good source of efficacy information (Maibach and Cotton, 1995; Cline and Young, 2004).

The fact that the photos and cartoons elicited almost the same levels of perceived severity and perceived response efficacy is surprising given photographs of a scene have been found to trigger not only more imagery but more vivid images than artistic versions of the same scene (Miller and Stoica, 2003). However, the invisible nature of mental illness and the fact that cartoons make visible symptoms that might be hard to imagine could account for perceived severity and response efficacy scores that were equivalent to those elicited by photos. 
Although previous studies have found that familiarity with mental illness leads to less social distancing behavior (Corrigan et al., 2001; Link \& Cullen, 1986), in this study familiarity with mental illness did not play a moderating role with regard to this variable. However, the affect variable connectedness acted as a mediator between image type and social distance. Specifically, those in the photo condition connected more with the protagonist in the story than those in the text condition, and this connectedness in turn led to lower social distancing behavior. This in line with research that has found that the more the viewer/reader identifies with a character in a movie about mental illness, the more relevant he/she perceives the story to be, and the less social distancing behavior he/she exhibits (Caputo \& Rouner, 2011).

Photos were more effective at leading to mental health policy support than text alone. (Also, compared to cartoon and text, means for the photo condition were the highest.) It's possible that this was so because the photographs raised the perceived importance of the issue (Wanta, 1988). Further, this effect was mediated through identification and connectedness with the story's protagonist. This finding supports previous research that shows that those who identify with the mentally ill are more likely to support government spending for mental health (McSween, 2002). This effect was, however, not moderated by political ideology, as found in the case of other stigmatizing diseases such as obesity (for example, Gollust, Lantz, \& Ubel, 2009).

There was no significant difference between the groups with regard to perceived responsibility beliefs; however, the mean for the photo condition was the lowest (low values indicate less attribution of responsibility for mental illness). Given that photographs prompted identification and connectedness with the story protagonist, this 
outcome is logical. There was no significant difference between the groups with regard to coercion-segregation intention - all indicated very low coercion-segregation intentions. Taking into consideration this sample's high familiarity with mental illness, this outcome is not surprising. Also, in retrospect, it's possible that the wording of the items in the coercion-segregation measure (for example, "If I were in charge of Eliza's treatment, I would force her to live in a group home.") might have elicited socially desirable responses.

Participants in the photo condition were more like to share the story than those in the cartoon or text condition. This effect was mediated by connectedness and identification with the story's protagonist. This is a logical finding given that the sharing of stigma appeals/messages is predicted by a negative response to the message (Smith, 2012), and so by corollary, the sharing of an anti-stigma message (which is how the stories in this study can be characterized) should be triggered by positive affect. Further, according to prior research on message sharing, messages that are more emotionally evocative are more likely to go viral (Berger, 2014; Berger and Milkman, 2012). Participants in the photo condition reported high identification and connectedness with the story protagonist. It is probable that these emotions were heightened by the photographs in the story, which several participants described as "humanizing." Emotional arousal leads to sharing of that emotion "because emotion sharing has both intrapersonal and interpersonal benefits, such as sense-making of the emotional experience and establishment (or strengthening) of social bonds (Rimé, 2009, as cited by Kim, 2015, p. 514). Story sharing was also higher for those who had higher familiarity with mental illness. 
Those in the cartoon condition reported less intention to disseminate the message compared to those in the photo condition. This is surprising because the use of cartoons in news narratives can be considered a novel form of storytelling, and novel messages have "high social currency and make for good conversation material" (Berger, 2013, 2014, as cited by Kim, 2015, p. 515). People are more likely to disseminate novel messages (Berger and Milkman, 2012). However, this was not the case in this study. It's possible that this is because photographs of people with mental illness are still a rarity in news media, and therefore, the photographs elicited greater attention.

For participants who viewed the story on depression, there was no significant difference between the text vs. photo groups or the text vs. cartoon groups with regard to donation behavior. However, there was a marginally significant difference between the cartoon and photo conditions, such that those in the cartoon condition were more likely to donate than those in the photo condition. This finding is partly in line with previous research that shows that people are more willing to make a charitable contribution when the appeal has a picture (Perrine and Heather, 2000). Further, mental images and attention prompt affective feelings toward people in distress (Slovic, 2007), and that the affective response is essential to helping behavior (Harbaugh, Mayr, \& Burghart, 2007; Moll et al., 2006; Genevsky, Västfjäll, Slovic, Knutson, 2013). Affective responses motivate behavior (Zajonc, 1980), such as decision-making (Loewenstein and Lerner, 2003)), and they make information meaningful (Cabanac M (1992)). It's not clear, though, why those in the cartoon condition were more likely to donate than those in the photo condition, given that photographs, not cartoons elicited higher levels of connectedness.

With regard to recall, there was no significant difference between the three 
groups. Only a small number of people $(\mathrm{n}=5)$ in the cartoon condition answered the recall questions correctly compared to those in the photo condition. This is surprising given that one of the purported advantages of comics is that the combination of text and images makes the information more memorable. It might be that in this study the cartoons offered no advantage because more than half of the participants reported to be highly familiar with mental illness and so because of their prior knowledge of mental disorders, could have found the recall questions easy to answer.

For the depression condition, the open-ended responses reflected the quantitative findings. Several participants in the text-alone condition indicated that they felt sympathy toward the story's protagonist and used words like "sympathy" and "concern" to describe their feelings. Several said that they could relate with the protagonist's struggles because either they themselves had experienced similar symptoms or had a friend or partner experience the disease. This corroborates the finding that a little more than $50 \%$ of the sample for this study had high familiarity with mental illness. Based on the responses to the photo condition and considering them together with the quantitative findings for the photo condition, it appears that the photographs heightened participants' feelings of empathy and led to stronger identification and connectedness. From this it can be inferred, that even though depression is a common disease and has low stigma (given that people feel comfortable disclosing that they themselves or someone close to them has experienced the disease), the addition of photographs to textual information still significantly increases the empathic response to people with the illness.

With regard to the open-ended responses to the cartoon images, most participants reported liking the images, described them as "accurate," "interesting," "informative," 
and "creative." They said that the images did a good job of "showing" the symptoms of depression and offered a better understanding of depression. However, participants did not describe the images as "humanizing," "touching," "empathetic"—words that were used to describe the photographs. It appears that although the cartoons did evoke identification and sympathy to some extent, they mainly served an explanatory function. Some participants also noted that the cartoons were "inappropriate" and "distracted from the seriousness of the story." This ties in with the quantitative findings, which showed that the photos were more effective at evoking connectedness with the story's protagonist.

Bipolar condition. There were no significant differences between the three groups - text, photo, and cartoon - in the bipolar condition with regard to any of the dependent variables. Also, there was no consistent pattern of results.

There were no significant differences between the three conditions with regard to perceived credibility and story enjoyment. For perceived credibility, the mean for the text condition $(M=18.7)$ was the highest and that for the cartoon condition $(M=18.09)$ was the lowest. However, both values are at the 6-point mark on a 7-point scale for perceived credibility (1, low credibility; 7 , high credibility), indicating that people in both conditions felt the story was highly credible. A similar pattern was observed for story enjoyment, that is, highest values for enjoyment in the text condition and lowest values in the cartoon condition. Again, the means indicated that participants in all three conditions felt that the story was enjoyable $(\mathrm{M}($ photo $)=5.59, \mathrm{M}($ text $)=5.75, \mathrm{M}($ cartoon $)=5.49))$.

With regard to identification, again, there were no significant differences between the three conditions. However, unlike the findings for the depression condition, here, the 
mean for the photo condition was the lowest and that for the text condition was the highest $(\mathrm{M}($ photo $)=24.6, \mathrm{M}($ text $)=25.56, \mathrm{M}($ cartoon $)=25.12))$. This might be because the text was found to be well-written and so the photographs had no added effect. It could also be that given that bipolar disorder is a relatively less frequently diagnosed illness compared to depression, participants may not have had personal experience with the illness. So they would have had to rely on their recollection of images of the severely mentally ill typically found in media, such as people with "unkempt hair," "tattered clothing," and "staring eyes" (Cross, 2004). Because the photographs in the stimuli did not resemble such representations, it is possible that they caused confusion rather than identification. Indeed, the word "normal" to describe the protagonist came up several times in the open-ended responses to the photographs. Most participants also commented on how "attractive" and "pretty" and "happy" the protagonist looked.

With regard to pity and connectedness, there were no significant differences between the three groups, but means for the cartoon conditions were the highest. This might be because the cartoons made the symptoms of the disorder visible and therefore, more understandable. This might also be because the cartoons were perceived as "nonthreatening" (McAllister, 1992) and so reduced the relatively high stigma (compared with depression) associated with bipolar disorder.

A different pattern was observed with regard to prejudicial feelingsthe lowest prejudicial feelings $(\mathrm{M}=10.13)$ and the photo $(\mathrm{M}=10.67))$ and cartoon conditions the highest $(M=10.69)$. However, again, it is important to note that the means indicate that participants in all three groups had very low prejudicial feelings.

There were no significant differences between the three conditions with regard to 
perceived severity and perceived response efficacy beliefs. The means for the three conditions were similar and indicated high perceived severity and high perceived response efficacy beliefs. Further, there were no significant differences between the means for the photo and cartoon conditions for perceived responsibility beliefs, and the means indicated low perceived responsibility. (The mean for the text condition could not be computed for this scale variable because, as explained earlier, the items had low reliability.)

With regard to stigmatizing behavioral intentions and behavior, there were no significant differences between the three groups with regard to coercion-segregation. The mean for the cartoon condition was the lowest, indicating low intention to coercesegregate. However, it is important to note that the means indicate that participants in all three groups had very low coercion-segregation intentions. With regard to mental health policy support, there were no significant differences between the three groups, and the means for all three groups indicated high mental health policy support. With regard to social distancing behavioral intentions, the means for the three groups were similar and indicated low intention to exercise socially distancing behavior. With regard to dissemination likelihood, there were no significant differences between the groups and the means indicated neutral intention to disseminate the message. Finally, there were no significant differences between the groups with regard to donation behavior.

The open-ended responses partially reflect these results in that participants in the cartoon condition described the images as "humanizing" and "moving" and helped them "empathize" and feel "compassion." These are opposite to the findings in the depression condition. This is intriguing. Given that the text and images for the depression and 
bipolar conditions were different, it is, unfortunately, not possible to compare the conditions to arrive at an explanation that would shed light on these opposing outcomes. It can be hypothesized, however, that the photos in the depression condition did a better job capturing the symptoms compared to the cartoon illustrations because depression is a relatively less complicated disease than bipolar disorder and also less invisible than bipolar disorder. Also, given the high prevalence of depression in the United States, most participants must have encountered someone with depression in their daily lives and based on the open-ended responses, it seems that the photographs in the story corroborated their personal experience with people with depression. The lack of effectiveness of the photographs in the bipolar condition might be because the photographs could not capture the complicated, severe, and invisible nature of bipolar disorder, whereas the cartoons could. It's not clear, though, why some participants felt that the cartoon illustrations were inappropriate for the depression condition, while none of the participants who were exposed to the cartoon version of the bipolar condition expressed this criticism. However, according to McNicol and Weaver (2013), such inconsistent findings are to be expected given the complicated nature of the comics medium. They argue that responses to comics will always be different because "the rhetoric of comics can be unstable and might support negative stereotypes or narratives in certain conditions" (p. 224). Further studies need to be done to tease out the processes underlying the different effects of comics in different conditions.

\section{Limitations and Future Studies}

The overarching goal of this study was to test the effects of the comics medium compared to the photographic medium in order to test the purported cognitive and 
emotional benefits of comics. For example, Squier (2008, p. 130) writes, “comics can reveal unvoiced relationships, unarticulated emotions, unspoken possibilities, and even unacknowledged alternative perspectives."

However, it can be argued that the study design doesn't capture the complexity of the comics medium as it is focused on testing the effect of only one aspect of the comics medium: cartoons — simplified images, devoid of details, with thought bubbles and that employ visual storytelling devices such as visual metaphors and personification. A story about mental illness with just text (control) was compared with the same story with text as well as photographs (treatment) as well as the same story with text and cartoons (treatment). Indeed, the lack of significant results in the bipolar condition of the experiment might be because the "comics" manipulation was too subtle, and consequently, might have led to type II errors. However, to limit confounding factors between the conditions in this study and to ensure as much equivalence as possible between the treatment conditions, the cartoons in the study were based on the photographs and were as similar as possible to the photographs. Future studies might test the effects of other comics elements, such as frame sequences, changes in font size to convey different emotions, and close-up shots versus long shots, as well as the effects of different styles of comics. In future studies, it might also be worthwhile to examine the effect of the comics medium as a whole instead of its composite parts/elements.

Reading comics is a cognitively demanding endeavor as readers have to make sense of not only text but also images (McCloud, 1993; McNicol \& Weaver, 2013; Squier, 2008). Comics are "highly complex compositions that require well-developed literacy" (McNicol \& Weaver, 2013, p. 217). This study aimed to test understanding of 
comics by measuring recall; however, the results revealed no significant differences between the conditions. This might be because the recall questions were too simple. The recall questions were not pre-tested for difficulty, and this is a limitation that could be rectified in future studies. In future studies it would be useful to measure familiarity with the comics medium to see whether it moderates effects. Finally, the study aimed to determine how style of processing moderates response to images in stories. However, because of scale reliability problems, this question could not be investigated. In future studies, it would be useful to use another measure of style of processing.

This study provokes a number of intriguing questions on the effects of comics, for example, can narratives on mental illness illustrated with cartoons reduce self-stigma? Is it ethical to use cartoons to depict certain illnesses? Can humor as an emotional response to comics narratives mediate anti-stigma behavioral intentions and behaviors? Which types of visual metaphors - a visual rhetorical device commonly used in comicstranslate across cultures? Such questions will provide fertile ground for further research.

\section{Theoretical and Practical Significance}

Comics are widely used in health communication messages, and practitioners and scholars of graphic medicine (the use of comics to tell stories about illness) argue that comics, by virtue of their unique characteristics, afford advantages that other media cannot. This study investigated this claim by examining the effects of different types of images - photographs and cartoons - in the context of a stigmatized disease, namely, mental illness. The findings contribute to several areas of research: the iconography of illness, stigma communication, and narrative persuasion.

How an illness is depicted influences how it is perceived; this in turn can affect 
how people with the disease are treated (Williams, 2011). Indeed, this study found that photographic illustrations of an individual's illness experience versus cartoon illustrations are perceived differently and have different effects in different contexts. Specifically, photographs accompanying a narrative on depression (a common and relatively less stigmatized disease) were perceived as "humanizing" and evoked identification and connectedness, which in turn led to prosocial behavioral intentions; cartoons depicting the same disease did not have this effect. On the other hand, cartoons accompanying a narrative on bipolar disorder (a relatively less common and more stigmatized disease) were described as evoking "empathy," whereas photographs depicting the same disease did not elicit this response.

This finding contributes to our understanding of stigma communication by illuminating the relationship between disease visibility and stigma. Studies have found that people with visible stigmatizing disorders are more stigmatized than concealable stigmatizing disorders. However, this study finds that there is more nuance to this relationship. This study finds that by helping the reader see the internal world of an individual with an invisible illness through the use of rhetorical visual devices, the comics medium can help readers better understand the illness compared to photographs or no imagery and empathize with the sufferer (Williams, 2011).

The study contributes to the literature on narrative persuasion by examining the effects of a unique type of visual narrative that has been used for decades - comics. Although a few previous studies have examined the effects of comics, this study is methodologically unique in that instead of examining the effect of the medium as a whole, it reduces the number of potentially confounding variables by adopting a 
controlled experimental design to delineate the effect of a particular characteristic of comics: cartoons. This study is also unique in that unlike previous studies of comics that focus on the medium's cognitive and educational effects, this study applies the principles of narrative persuasion to investigate the emotional and behavioral effects of cartoons. The study also shows that while images can enhance the persuasive potential of illness narratives compared to text-only narratives, some types of images are more effective than others, and this effectiveness depends on contextual factors such as the degree of visibility of the illness.

Finally, the study has practical implications for design of anti-stigma mental health campaigns and news stories. Cartoons should not be used indiscriminately in mental health campaigns as it appears that under certain circumstances, photographs might be more effective than cartoons. Comics journalists believe that the comics medium can help tell stories more thoroughly and effectively. According to Symbolia (a former graphic journalism magazine) editor Erin Polgreen, "Comics work well for journalism because they are able to convey multiple complicated layers of information in a package that is a little bit easier to digest than, say, a series of very, very intense photographs from a war zone or an article of 10,000 words" (Alverson, 2013). However, this conjecture needs to be empirically tested. Finally, comics are believed to have tremendous potential in patient education and physician-patient communication. However, whether comics are more effective at educating patients about diseases compared to other visual images and whether comics help physicians empathize with the patient experience need to be investigated. To use comics strategically in health communication, it is important to understand its advantages and limitations. 


\section{References}

Alesandrini, K. L. (1984). Pictures and adult learning. Instructional Science, 13(1), 6377.

Alverson, B. (2013, February 23). Interview: Symbolia Editor Erin Polgreen on Graphic Journalism for the Tablet. Good E Reader. Retrieved from http://goodereader.com/blog/interviews/interview-symbolia-editor-erin-polgreenon-graphic-journalism-for-the-tablet

Amazon Mechanical Turk. (n.d.). Retrieved from https://www.mturk.com/mturk/welcome

Angermeyer, M. C., Matschinger, H., Link, B. G., \& Shomerus, G. (2014). Public attitudes regarding individual and structural discrimination. Two sides of the same coin? Social Science and Medicine, 103, 60-66

Appelman, A., \& Sundar, S. S. (2015). Measuring Message Credibility Construction and Validation of an Exclusive Scale. Journalism \& Mass Communication Quarterly, 1077699015606057.

Arkar, H., \& Eker, D. (1994). Effect of psychiatric labels on attitudes toward mental illness in a Turkish sample. International Journal of Social Psychiatry, 40(3), 205-213.

Baddeley, A. D. (1992). Human memory. Boston: Allyn \& Bacon.

Barry, C. L., McGinty, E. E., Pescosolido, B. A., \& Goldman, H. H. (2014). Stigma, discrimination, treatment effectiveness, and policy: public views about drug addiction and mental illness. Psychiatric Services, 65(10), 1269-1272.

Behrend, T. S., Sharek, D. J., Meade, A. W., \& Wiebe, E. N. (2011). The viability of crowdsourcing for survey research. Behavior research methods, 43(3), 800-813.

Berger, J., \& Milkman, K. L. (2012). What makes online content viral?. Journal of marketing research, 49(2), 192-205.

Berger, J. (2014). Word of mouth and interpersonal communication: A review and directions for future research. Journal of Consumer Psychology, 24(4), 586-607.

Berinsky, A. J., Huber, G. A., \& Lenz, G. S. (2012). Evaluating online labor markets for experimental research: Amazon. com's Mechanical Turk. Political Analysis, 20(3), 351-368. 
Block, L. G., \& Keller, P. A. (1998). Beyond protection motivation: An integrative theory of health appeals. Journal of Applied Social Psychology, 28(17), 15841608.

Boer, H., Huurne, E. T., \& Taal, E. (2006). Effects of pictures and textual arguments in sun protection public service announcements. Cancer Detection and Prevention, $30,432-438$.

Bos, A. E., Pryor, J. B., Reeder, G. D., \& Stutterheim, S. E. (2013). Stigma: Advances in theory and research. Basic and Applied Social Psychology, 35(1), 1-9.

Cabanac, M. (1992). Pleasure: the common currency. Journal of theoretical Biology, 155(2), 173-200.

Caputo, N. M., \& Rouner, D. (2011). Narrative processing of entertainment media and mental illness stigma. Health communication, 26(7), 595-604.

Carney, R. N., \& Levin, J. R. (2002). Pictorial illustrations still improve students' learning from text. Educational psychology review, 14(1), 5-26.

Chaiken, S. (1980). Heuristic versus systematic information processing and the use of source versus message cues in persuasion. Journal of personality and social psychology, ), 752.

Chandler, P., \& Sweller, J. (1991). Cognitive load theory and the format of instruction. Cognition \& Instruction, 8, 293-332.

Chang, C. (2008). Increasing mental health literacy via narrative advertising. Journal of Health Communication: International Perspectives, 13(1), 37-55.

Chang, C. (2013). Seeing is believing: The direct and contingent influence of pictures in health promotion advertising. Health communication, 28(8), 822-834.

Chaudoir, S. R., Earnshaw, V. A., \& Andel, S. (2013) "Discredited" versus "Discreditable": understanding how shared and unique stigma mechanisms affect psychological and physical health disparities. Basic and Applied Social Psychology, 35(1), 75-87.

Childers, T. L., Houston, M. J., \& Heckler, S. E. (1985). Measurement of individual differences in visual versus verbal information processing. Journal of Consumer Research, 12(2), 125-134.

Cohen, J. (2001). Defining identification: A theoretical look at the identification of audiences with media characters. Mass communication \& society, 4(3), 245-264. 
Colin, P., Chauvet, F., \& Viennot, L. (2002). Reading images in optics: Students' difficulties and teachers' views. International Journal of Science Education, 24, 313-332. doi: 10.1080/09500690110078923

Corrigan, P., Markowitz, F. E., Watson, A., Rowan, D., \& Kubiak, M. A. (2003). An attribution model of public discrimination towards persons with mental illness. Journal of health and Social Behavior, 162-179.

Cross, S. (2004). Visualizing madness mental illness and public representation. Television \& New Media, 5(3), 197-216.

David, P. (1998). News Concreteness and Visual-Verbal Association Do News Pictures Narrow the Recall Gap Between Concrete and Abstract News?. Human Communication Research, 25(2), 180-201.

Delp, C., \& Jones, J. (1996). Communicating information to patients: the use of cartoon illustrations to improve comprehension of instructions. Academic Emergency Medicine, 3(3), 264-270.

Dwyer, F. M. (1978). Strategies for Improving Visual Learning. State College, Pa: Learning Services.

Dwyer, F. M. (1982-83). "The program of systematic evaluation - A brief review," International Journal of Instructional Media 10:23 38.

Eisner, W. (2008). Graphic Storytelling and Visual Narrative (Will Eisner Instructional Books). WW Norton \& Company.

Ericsson, K. A., \& Kintsch, W. (1995). Long-term working memory. Psychological Review, 102, 211-245. doi:10.1037/0033-295X.102.2.211

Faul, F., Erdfelder, E., Buchner, A., \& Lang, A. G. (2009). Statistical power analyses using G* Power 3.1: Tests for correlation and regression analyses. Behavior research methods, 41(4), 1149-1160.

Fies, B. (2006). Mom's cancer. New York: Abrams Image.

Fisher, M., \& Baum, F. (2010). The social determinants of mental health: implications for research and health promotion. Australian and New Zealand Journal of Psychiatry, 44(12), 1057-1063.

Forney, E. (2012). Marbles: Mania, Depression, Michelangelo, and Me: A Graphic Memoir. Penguin. 
Genevsky, A., Västfjäll, D., Slovic, P., \& Knutson, B. (2013). Neural underpinnings of the identifiable victim effect: Affect shifts preferences for giving. The Journal of Neuroscience, 33(43), 17188-17196.

Gibson, R., \& Zillmann, D. (2000). Reading between the photographs: The influence of incidental pictorial information on issue perception. Journalism \& Mass Communication Quarterly, 77, 355-366.

Glaser, B., \& Strauss, A. (1967). The discovery ofgrounded theory. London: Weidenfeld and Nicholson, 24(25), 288-304.

Gluibizzi, A. (2007). The aesthetics and academics of graphic novels and comics. Art Documentation, 26(1), 28-30.

Goffman, E. (1986). Stigma: notes on the management of spoiled identity. New York: Simon \& Schuster.

Gollust, S. E., Lantz, P. M., \& Ubel, P. A. (2009). The polarizing effect of news media messages about the social determinants of health. American Journal of Public Health, 99(12), 2160-2167.

Green, M. C., \& Brock, T. C. (2000). The role of transportation in the persuasiveness of . Journal of personality and social psychology, 79(5), 701.

Green, M. C., Brock, T. C., \& Kaufman, G. E. (2004). Understanding media enjoyment: The role of transportation into narrative worlds. Communication Theory, 14, 311327.

Green, M. J., \& Myers, K. R. (2010). Graphic medicine: use of comics in medical education and patient care. $B M J, 340$.

Greer, J. D., \& Gosen, J. D. (2002). How much is too much? assessing levels of digital alteration of factors in public perception of news media credibility. Visual Communication Quarterly, 9(3), 4-13.

Gruber, D., \& Dickerson, J. A. (2012). Persuasive images in popular science: Testing judgments of scientific reasoning and credibility. Public Understanding of Science, 21(8), 938-948.

Hansen, B. (2004). Medical history for the masses: how American comic books celebrated heroes of medicine in the 1940s. Bulletin of the History of Medicine, 78(1), 148-191.

Harbaugh, W. T., Mayr, U., \& Burghart, D. R. (2007). Neural responses to taxation and voluntary giving reveal motives for charitable donations. Science, 316(5831), $1622-1625$. 
Hayes, A. F. (2013). Introduction to mediation, moderation, and conditional process analysis: A regression-based approach. Guilford Press.

Hinyard, L. J., \& Kreuter, M. W. (2007). Using narrative communication as a tool for health behavior change: A conceptual, theoretical, and empirical overview. Health Education and Behavior, 34, 777-792.

Holmes, E. P., Corrigan, P. W., Williams, P., Canar, J., \& Kubiak, M. A. (1999). Changing attitudes about schizophrenia. Schizophrenia bulletin, 25(3), 447.

Hosler, J., \& Boomer, K. B. (2011). Are Comic Books an Effective Way to Engage Nonmajors in Learning and Appreciating Science? 1. CBE-Life Sciences Education, 10(3), 309-317.

Huh, H. J. L. (1993). The Effect of Newspaper Picture Size on Readers' Attention, Recall, and Comprehension of Stories.

Johnstone, M. (2005). I had a black dog: his name was depression. Pan Austra

Jones, E. E. (1984). Dimensions of Stigma.

Jee, B. D., \& Anggoro, F. K. (2012). Comic cognition: exploring the potential cognitive impacts of science comics. Journal of Cognitive Education and Psychology, 11(2), 196-208.

Kahan, D. M., Braman, D., Monahan, J., Callahan, L., \& Peters, E. (2010). Cultural cognition and public policy: The case of outpatient commitment laws. Law and Human Behavior, 34(2), 118-140.

Khoii, R., \& Forouzesh, Z. (2010). Using comic strips with reading texts: are we making a mistake. Literacy Information and Computer Educational Journal, 1(3), 168177.

Koedinger, K. R., \& Nathan,M. J. (2004). The real story behind story problems: Effects of representations on quantitative reasoning. Journal of the Learning Sciences, 13, 29-164. doi: 10.1207/s15327809j1s13021

Kim, H. S. (2015). Attracting Views and Going Viral: How Message Features and NewsSharing Channels Affect Health News Diffusion. Journal of Communication, 65(3), 512-534.

Kinzer, Charles K., et al. "Examining the effects of text and images on story comprehension: An eye-tracking study of reading in a video game and comic book." 61st Yearbook of the Literacy Research Association (2012): 259. 
Levin, J. R., and Mayer, R. E. (1993). Understanding illustrations in text. In Britton, B. K., Woodward, A., and Brinkley,M. (eds.), Learning from Textbooks, Erlbaum, Hillsdale, NJ, pp. 95-113.

Lin, S. F., Lin, H. S., Lee, L., \& Yore, L. D. (2014). Are Science Comics a Good Medium for Science Communication? The Case for Public Learning of Nanotechnology. International Journal of Science Education, Part B, (ahead-ofprint), 1-19.

Link, B. G., \& Cullen, F. T. (1986). Contact with the mentally ill and perceptions of how dangerous they are. Journal of Health and Social Behavior, 289-302.

Link, B. G., Cullen, F. T., Frank, J., \& Wozniak, J. F. (1987). The social rejection of former mental patients: Understanding why labels matter. American journal of Sociology, 1461-1500.

Link, B. G., \& Phelan, J. C. (2001). Conceptualizing stigma. Annual review of sociology, $27,363-385$.

Link, B. G., \& Phelan, J. C. (2013). Labeling and stigma. In Handbook of the sociology of mental health (pp. 525-541). Springer Netherlands.

Linthout, W., \& Linthout, T. (2009). Years of the Elephant. Ponent Mon.

Maibach, E. W., \& Cotton, D. (1995). Moving people to behavior change: a staged social cognitive approach to message design.

Mayer, R. E. (2003). The promise of multimedia learning: using the same instructional design methods across different media. Learning and instruction, 13(2), 125-139.

McAllister, M. P. (1992). Comic books and AIDS. The Journal of Popular Culture, 26(2), 1-24.

McCloud, S. (1993). Understanding comics: The invisible art. Northampton, Mass.

Mechanial Turk Concepts. (n.d.) Retrieved from http://docs.aws.amazon.com/AWSMechTurk/latest/RequesterUI/mechanical-turkconcepts.html

Moll, J., Krueger, F., Zahn, R., Pardini, M., de Oliveira-Souza, R., \& Grafman, J. (2006). Human fronto-mesolimbic networks guide decisions about charitable donation. Proceedings of the National Academy of Sciences, 103(42), 1562315628 .

Iyengar, S. (1991). Is anyone responsible? How television frames political issues. Chicago, IL: University of Chicago Press. 
Kogut, T., \& Ritov, I. (2005). The "identified victim" effect: An identified group, or just a single individual? Journal of Behavioral Decision Making, 18, 157-167. doi:10.1002/bdm.492

Lee, T. J., Cameron, L. D., Wunsche, B., \& Stevens, C. (2011). A randomized trial of computer-based communications using imagery and test information to alter representations of heart disease risk and motivate protective behaviour. British Journal of Health Psychology, 16, 72-91.

Levie, W. H., \& Lentz, R. (1982). Effects of text illustrations: A review of research. ECTJ, 30(4), 195-232.

Liu, J. (2004). Effects of comic strips on L2 learners' reading comprehension. TESOL quarterly, 38(2), 225-243.

Loewenstein, G., \& Lerner, J. S. (2003). The role of affect in decision making. Handbook of affective science, 619(642), 3.

Mason, L., Pluchino, P., Tornatora, M. C., \& Ariasi, N. (2013). An eye-tracking study of learning from science text with concrete and abstract illustrations. The Journal of Experimental Education, 81(3), 356-384.

Medley, S. , \& Haddad, H. (2011). The realism continuum, representation and perception. The International Journal of the Image, 1(2), 145-156.

MERÇ, A. P. D. A., \& Kampusu, Y. (2013). The effect of comic strips on efl reading comprehension. International Journal on New Trends in Education and Their Implications.

McNicol, S. (2014). Humanising illness: presenting health information in educational comics. Medical humanities, 40(1), 49-55.

McSween, J. L. (2002). The role of group interest, identity, and stigma in determining mental health policy preferences. Journal of Health Politics, Policy and Law, 27(5), 773-800.

Miller, D. W., \& Stoica, M. (2004). Comparing the effects of a photograph versus artistic renditions of a beach scene in a direct-response print ad for a Caribbean resort island: A mental imagery perspective. Journal of Vacation Marketing, 10(1), 1121.

Niederdeppe, J., Bu, Q., Borah, P., Kindig, D. A., \& Robert, S. A. (2008). Message design strategies to raise public awareness of social determinants of health and population health disparities. Milbank Quarterly, 86(3), 481-513. 
Niederdeppe, J., Shapiro, M. A., \& Porticella, N. (2011). Attributions of responsibility for obesity: Narrative communication reduces reactive counterarguing among liberals. Human Communication Research, 37(3), 295-323.

Niederdeppe, J., Kim, H. K., Lundell, H., Fazili, F., \& Frazier, B. (2012). Beyond counterarguing: Simple elaboration, complex integration, and counterelaboration in response to variations in narrative focus and sidedness. Journal of Communication, 62, 758-777. doi:10.1111/j.1460-2466.2012.01671.

Niederdeppe, J., Roh, S., \& Dreisbach, C. (2015). How Narrative Focus and a Statistical Map Shape Health Policy Support Among State Legislators. Health communication, 1-14.

Nyberg, A. K. (2010). How librarians learned to love the graphic novel. Graphic Novels and Comics in Libraries and Archives: Essays on Readers, Research, History, and Cataloging. Ed. Robert G. Weiner. Jefferson, NC: McFarland, 26-40.

Nyberg, A. K. (2013). Comics Code History: The Seal of Approval. Comic Book Legal Defense Fund, 30.

Oliver, M. B., Dillard, J. P., Bae, K., \& Tamul, D. J. (2012). The effect of narrative news format on empathy for stigmatized groups. Journalism \& Mass Communication Quarterly, 89(2), 205-224.

Paolacci, G., Chandler, J., \& Ipeirotis, P. G. (2010). Running experiments on amazon mechanical turk. Judgment and Decision making, 5(5), 411-419.

Peeck, J. (1993). Increasing picture effects in learning from illustrated text. Learn. Instruct. 3: 227-238.

Perrine, R. M., \& Heather, S. (2000). Effects of picture and even-a-penny-will-help appeals on anonymous donations to charity. Psychological Reports, 86(2), 551559.

Petersen, R. S. (2011). Comics, Manga, And Graphic Novels: A History of Graphic Narratives. ABC-CLIO.

Paivio, A. (1986). Mental representations: A dual coding approach. Oxford: England: Oxford University Press.

Pescosolido, B.A., Medina, T.R., Martin, J.K., and Long, S.J. (2013). The "backbone" of stigma: Identifying the global core of public prejudice associated with mental illness. Am J Public Health, 103, 853-860. 
Phelan, J. C., Link, B. G., Stueve, A., \& Pescosolido, B. A. (2000). Public conceptions of mental illness in 1950 and 1996: What is mental illness and is it to be feared? Journal of Health and Social Behavior, 41(2), 188-207.

Phelan, J. C., Link, B. G., \& Dovidio, J. F. (2008). Stigma and prejudice: One animal or two? Social Science and Medicine, 67, 358-367.

Pryor, J. B., \& Reeder, G. D. (2011). HIV-related stigma. In J. C. Hall, B. J. Hall \& C. J. Cockerell (Eds.), HIV/AIDS in the Post-HAART Era: manifestations, treatment, and Epidemiology (pp. 790-806). Shelton, CT: PMPHUSA.

Ramasubramanian, S. (2010). Television viewing, racial attitudes, and policy preferences: Exploring the role of social identity and intergroup emotions in influencing support for affirmative action. Communication Monographs, 77(1), 102-120.

Rand, D. G. (2012). The promise of Mechanical Turk: How online labor markets can help theorists run behavioral experiments. Journal of theoretical biology, 299, 172179.

Reeves, William C., Tara W. Strine, Laura A. Pratt, William Thompson, Indu Ahluwalia, Satvinder S. Dhingra, Lela R. McKnight-Eily, et al. 2011. "Mental Illness Surveillance Among Adults in the United States." Morbidity and Mortality Weekly Report, 60 (03), 1-32.

Reitberger, R. C., \& Fuchs, W. J. (1972). Comics: Anatomy of a mass medium. Studio Vista.

Rimé, B. (2009). Emotion elicits the social sharing of emotion: Theory and empirical review. Emotion Review, 1(1), 60-85.

Schneider, E. F. (2014). Quantifying and Visualizing the History of Public Health Comics. In iConference 2014 Proceedings (p. 995-997). doi:10.9776/14340

Sim, M. G., McEvoy, A. C., Wain, T. D., \& Khong, E. L. (2014). Improving health Professional's knowledge of hepatitis B using cartoon based learning tools: a retrospective analysis of pre and post tests. BMC medical education, 14(1), 244.

Sinha, I., Patel, A., Kim, F. S., MacCorkle, M. L., \& Watkins, J. F. (2011). Comic books can educate children about burn safety in developing countries. Journal of Burn Care \& Research, 32(4), e112-e117.

Squier, S. M. (2008). So long as they grow out of it: comics, the discourse of developmental normalcy, and disability. Journal of medical humanities, 29(2), 71-88. 
Smith, R. A. (2007). Language of the lost: An explication of stigma communication. Communication Theory, 17(4), 462-485.

Smith, R. A. (2012). An Experimental Test of Stigma Communication Content with a Hypothetical Infectious Disease Alert. Communication Monographs, 79(4), 522538.

Tal-Or, N., \& Cohen, J. (2010). Understanding audience involvement: Conceptualizing and manipulating identification and transportation. Poetics, 38(4), 402-418.

Tatalovic, M. (2009). Science comics as tools for science education and communication: a brief, exploratory study. Jcom, 8(4).

Taylor, J. (2000). Problems in Photojournalism: realism, the nature of news and the humanitarian narrative. Journalism studies, 1(1), 129-143.

Todman, L. (2011). The social determinants of mental health. The SES Indicator. Retrieved from http://www.apa.org/pi/ses/resources/indicator/2011/08/lynntodman.aspx

U.S. Department of Health and Human Services. (1999). Mental Health: A Report of the Surgeon General-Executive Summary. Rockville, MD: U.S. Department of Health and Human Services, Substance Abuse and Mental Health Services Administration, Center for Mental Health Services, National Institutes of Health, National Institute of Mental Health.

Varnum, R., \& Gibbons, C. T. (2001). The language of comics: Word and image. Jackson: University Press of Mississippi.

Walters, G., Sparks, B., \& Herington, C. (2007). The effectiveness of print advertising stimuli in evoking elaborate consumption visions for potential travelers. Journal of Travel Research, 46(1), 24-34.

Wanta, W. (1988). The effects of dominant photographs: An agenda-setting experiment. Journalism and Mass Communication Quarterly, 65(1), 107.

Wanta, W., \& Roark, V. (1993). Cognitive and Affective Responses to Newspaper Photographs.

Weiner, B. (1993). On sin versus sickness: A theory of perceived responsibility and social motivation. American Psychologist, 48(9), 957.

Weiner, B. (2006). Social motivation, justice, and the moral emotions: An attributional approach. Mahwah, NJ: Lawrence Erlbaum Associates. 
Welch Cline, R. J., \& Young, H. N. (2004). Marketing drugs, marketing health care relationships: A content analysis of visual cues in direct-to-consumer prescription drug advertising. Health Communication, 16(2), 131-157.

Wildavsky, A., \& Dake, K. (1990). Theories of risk perception: Who fears what and why?. Daedalus, 41-60.

Wileman, R. E. (1993). Visual Communicating. New Jersey: Educational Technology Publications.

Williams, I. (2011). Graphic medicine: how comics are revolutionizing the representation of illness. Hektoen International. A Journal of Medical Humanities.

Williams, K. (2005). The Case for Comics Journalism. Columbia Journalism Review, 43, $51-55$.

Witte, K. (1992). Putting the fear back into fear appeals: The extended parallel process model. Communications Monographs, 59(4), 329-349.

Wittrock, M. C. (1989). Generative processes of comprehension. Educational psychologist, 24, 345-376

Yang, L. H., Kleinman, A., Link, B. G., Phelan, J. C., Lee, S., \& Good, B. (2007). Culture and stigma: adding moral experience to stigma theory. Social science \& medicine, 64(7), 1524-1535.

Yeh, M. A., Jewell, R. D., \& Hu, M. Y. (2013). Stereotype processing's effect on the impact of the myth/fact message format and the role of personal relevance. Psychology \& Marketing, 30(1), 36-45.

Young, R., Subramanian, R., \& Hinnant, A. (2015). Stigmatizing Images in Obesity Health Campaign Messages and Healthy Behavioral Intentions. Health Education \& Behavior, 1090198115604624.

Zajonc, R. B. (1980). Feeling and thinking: Preferences need no inferences. American psychologist, 35(2), 151.

Zillmann, D. (1998). The psychology of the appeal of portrayals of violence. Why we watch: The attractions of violent entertainment, 179-211.

Zillmann, D., Gibson, R., \& Sargent, S. L. (1999). Effects of photographs in newsmagazine reports on issue perception. Media Psychology, 1(3), 207-228.

Zillmann, D., \& Brosius H. (2000). Exemplification in communication: The influence of case reports on the perception of issues. Mahwah, NJ: Lawrence Erlbaum Associates. 
Zillmann, D., Knobloch, S., \& Yu, H. S. (2001). Effects of photographs on the selective reading of news reports. Media Psychology, 3(4), 301-324. 


\title{
Appendix
}

\author{
Model Release Form
}

Thanks again for serving as the model for my fictitious feature stories on mental illness. As I explained, the stories will be used in a research study/studies, specifically, in an online experiment/experiments. Therefore, these visuals (photos and cartoon illustrations based on the photos) will be viewed by the study participants. At the end of the study, however, participants will be notified that the visuals/accompanying stories are fictitious. The visuals may also be presented at research conferences, might be published in academic journals, or other academic literature. The visuals will also be viewed by a graphic design artist who will be creating illustrations based on the photos.

Please indicate your consent to the above terms of usage of the visuals (photographs and cartoon illustrations) by signing below:

Signature

Date 


\section{VITA}

Roma Subramanian's academic background is in biology and journalism: She has a master's degree in life sciences from the University of Mumbai, India, and she completed a second master's in science and technology journalism from Texas A\&M University, College Station, Texas.

Before joining A\&M, Roma was a scientific manuscript editor for Cactus Communications in Mumbai, India. Her work involved checking scientific papers for errors in language and format and training new entrants to the editing team. Roma's media experience also includes science writing. While at A\&M, she worked as a science writer at the public relations department of the university's veterinary school.

Roma's research interest is in health communication, particularly, stigma communication. Research in the area of health stigma includes investigating, through indepth interviews with award-winning journalists, how mental illness news coverage in the United States can be improved; exploring how suicide is framed episodically and thematically by U.S. college newspapers and the effects of these frames on students' attributions about responsibility for suicide; determining what are the effects of antiobesity campaigns on anti-fat attitudes and health-related behavioral intentions; and examining how anti-stigma public service announcements on mental illness inadvertently contain stigmatizing messages. Her doctoral dissertation, builds on this stream of research by investigating the potential of health narratives in a graphics/comics format to reduce mental illness stigma.

Other research includes the impact of social and mobile media on health. For example, she has examined what social media use can tell us about emotional well-being, 
particularly, self-esteem, and what health professionals in Sri Lanka think are the challenges and opportunities to using mobile health (m-health) technology.

Finally, her professional background in science writing and editing has piqued my interest in science communication research. Studies include investigating how climate change news stories with a public health frame influence beliefs about the certainty of climate change and whether comments on these stories disrupt reception of the story message. 